\title{
Isotopic Consequences of Host-guest Interactions; Noncovalent Chlorine Isotope Effects
}

\author{
Agata Paneth ${ }^{1}$ and Piotr Paneth ${ }^{2 *}$ \\ 1. Department of Organic Chemistry, Faculty of Pharmacy, Medical University \\ of Lublin, Chodźki 4a, 20-093 Lublin, Poland \\ 2. Institute of Applied Radiation Chemistry, Faculty of Chemistry, Lodz \\ University of Technology, Żeromskiego 116, 90-924 Lodz, Poland,
}

\section{Contents}

1. Cartesian coordinates of the gas phase structures of host-guest complexes $\mathbf{1}, \mathbf{3}$, and $\mathbf{4}$,

2. Cartesian coordinates of the structures of host-guest complexes $\mathbf{2}$ in the continuum DSMO model,

3. Figure S1. Illustration of the Langevin dynamics calculations,

4. Cartesian coordinates of the QM/MM structures of host-guest complexes $\mathbf{1}$. 
Cartesian coordinates of the gas phase structures of host-guest complexes $\mathbf{1 , 3}$, and $\mathbf{4}$

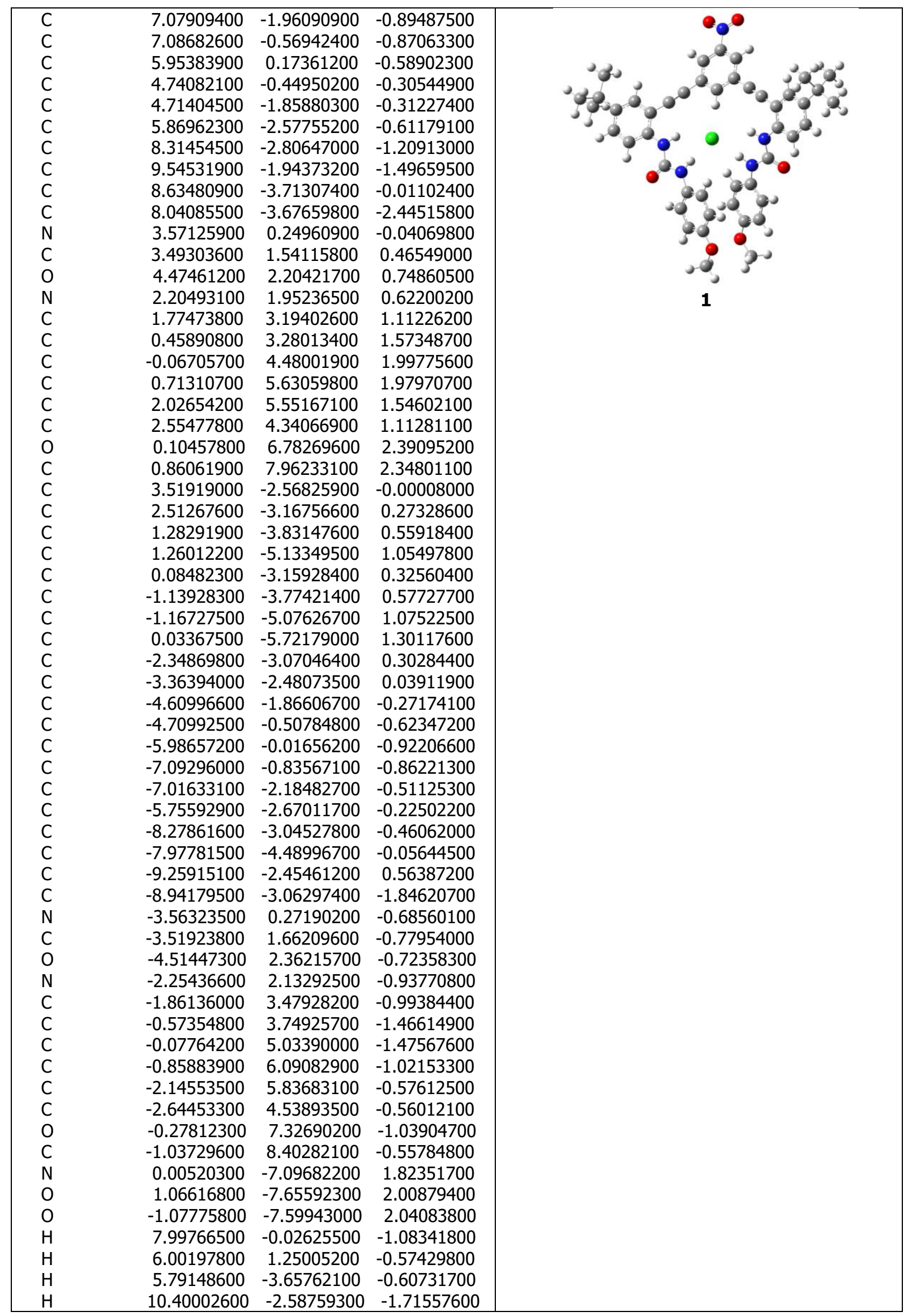




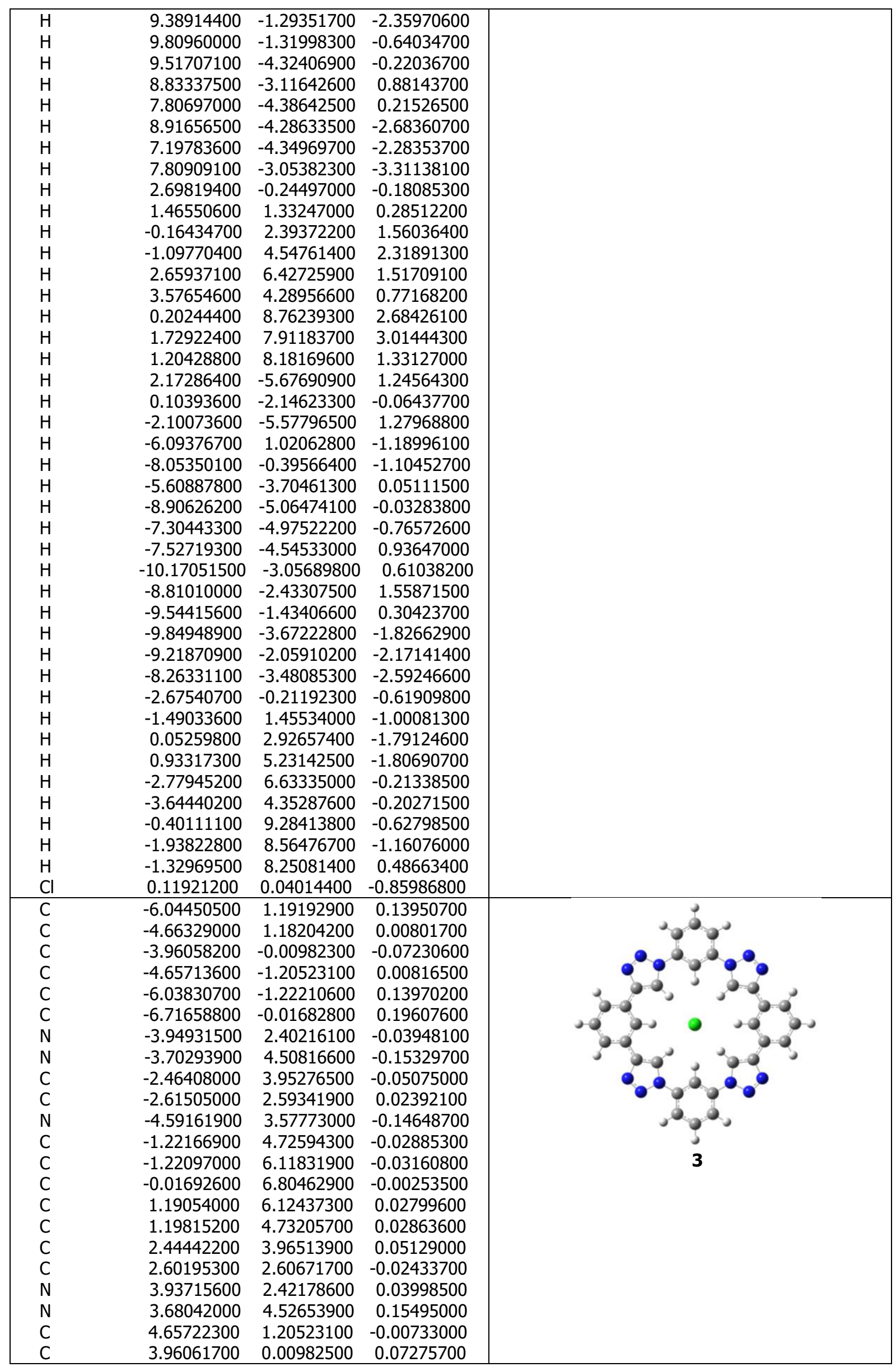




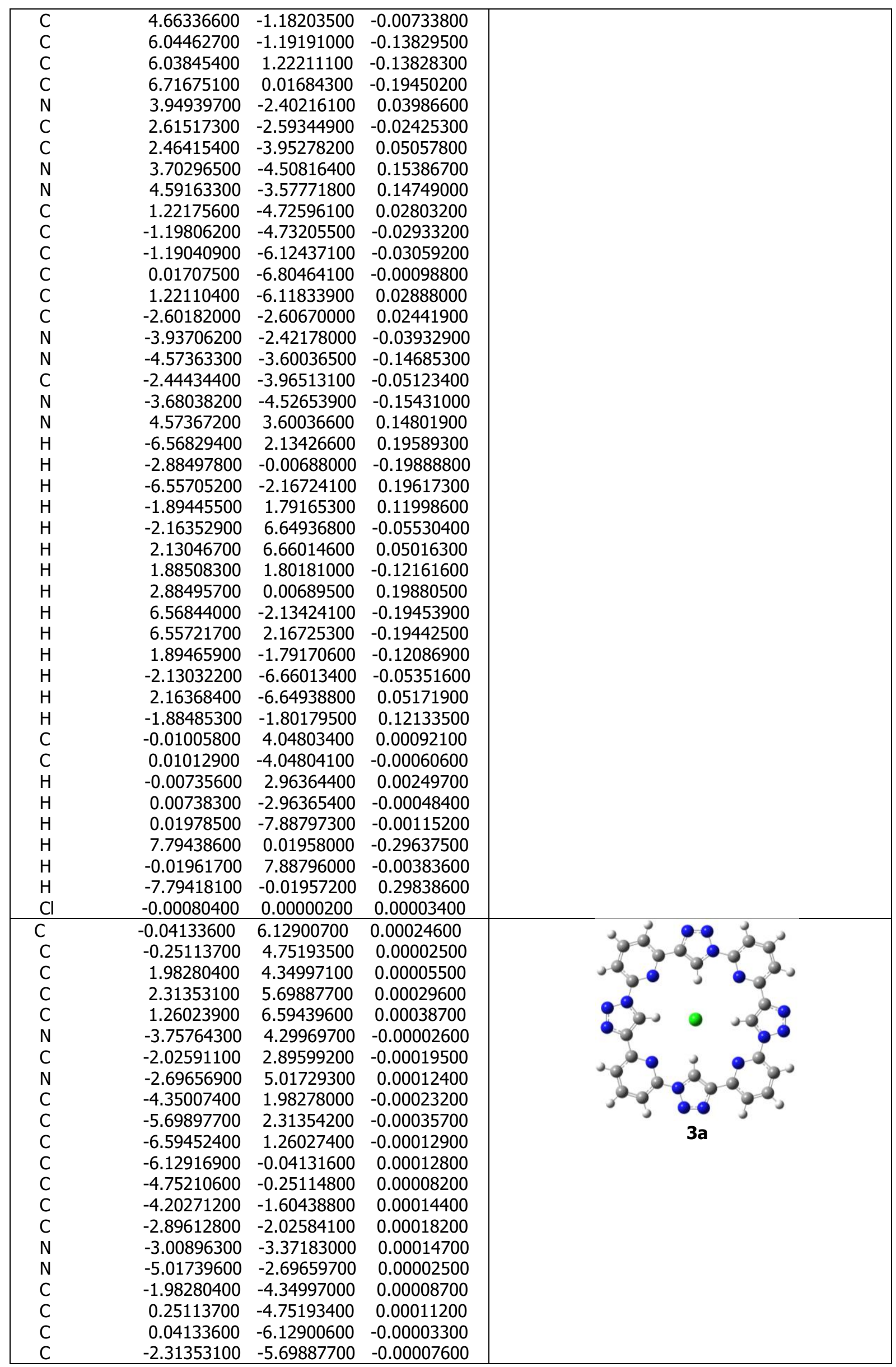




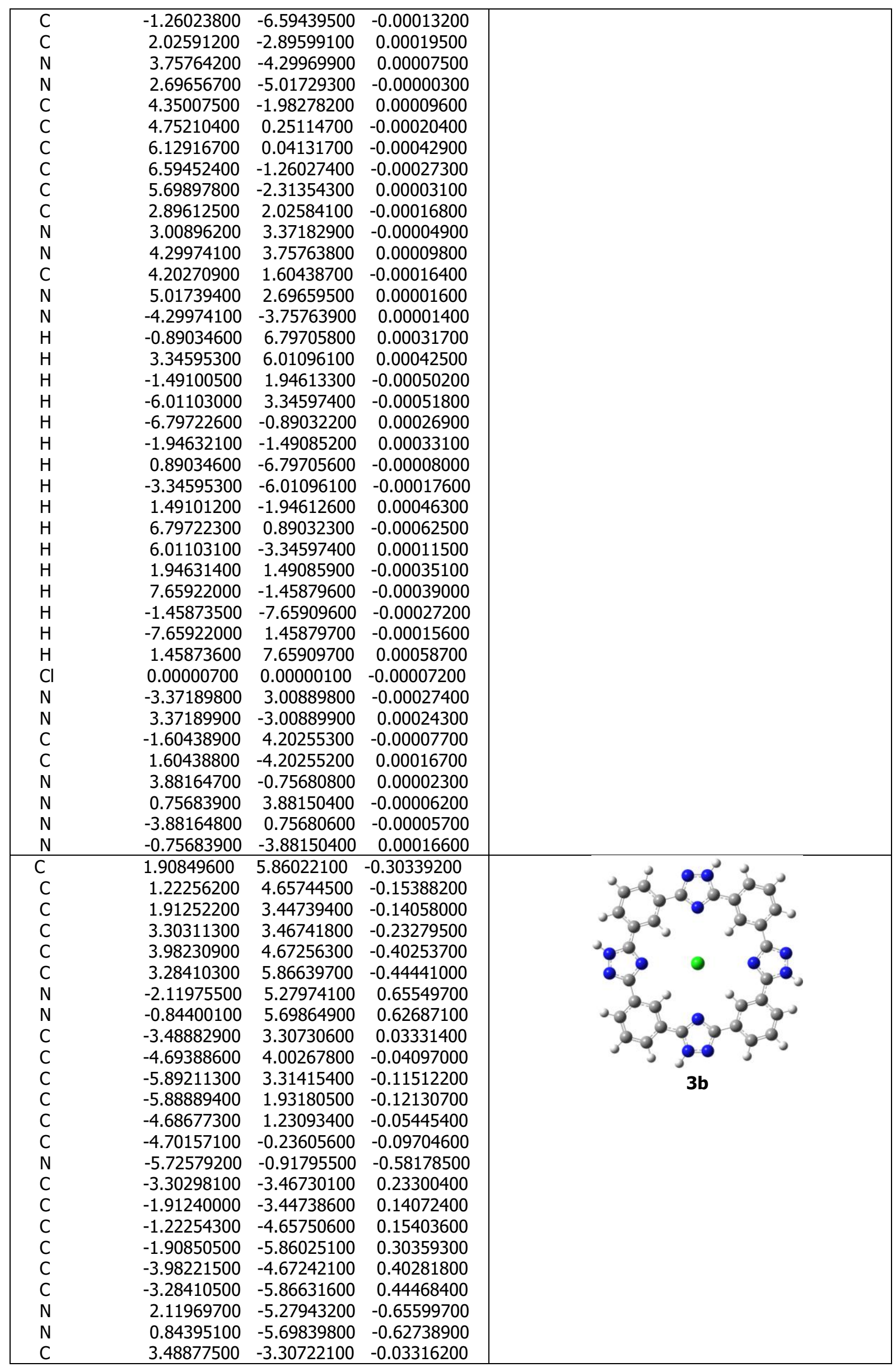




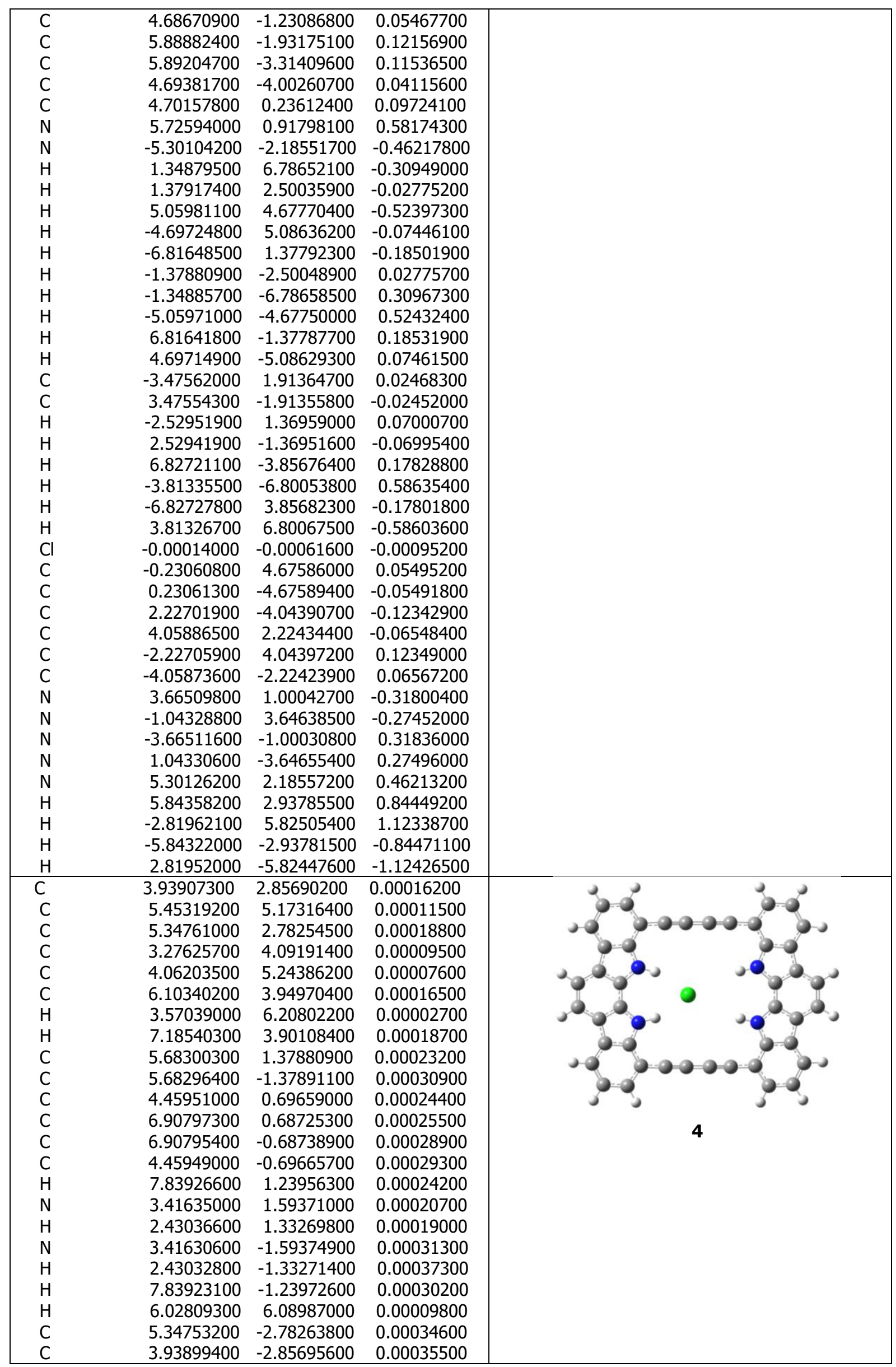




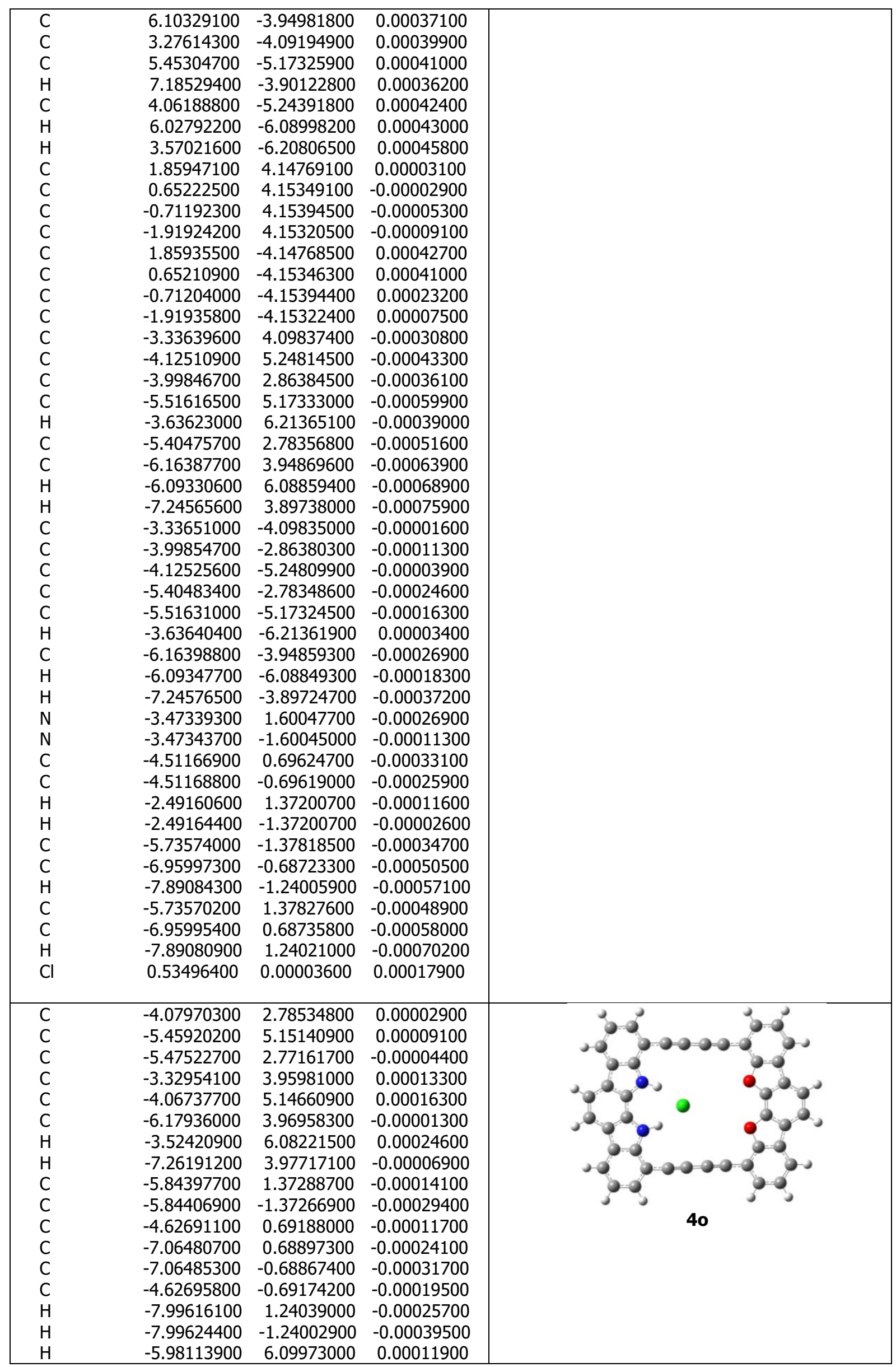




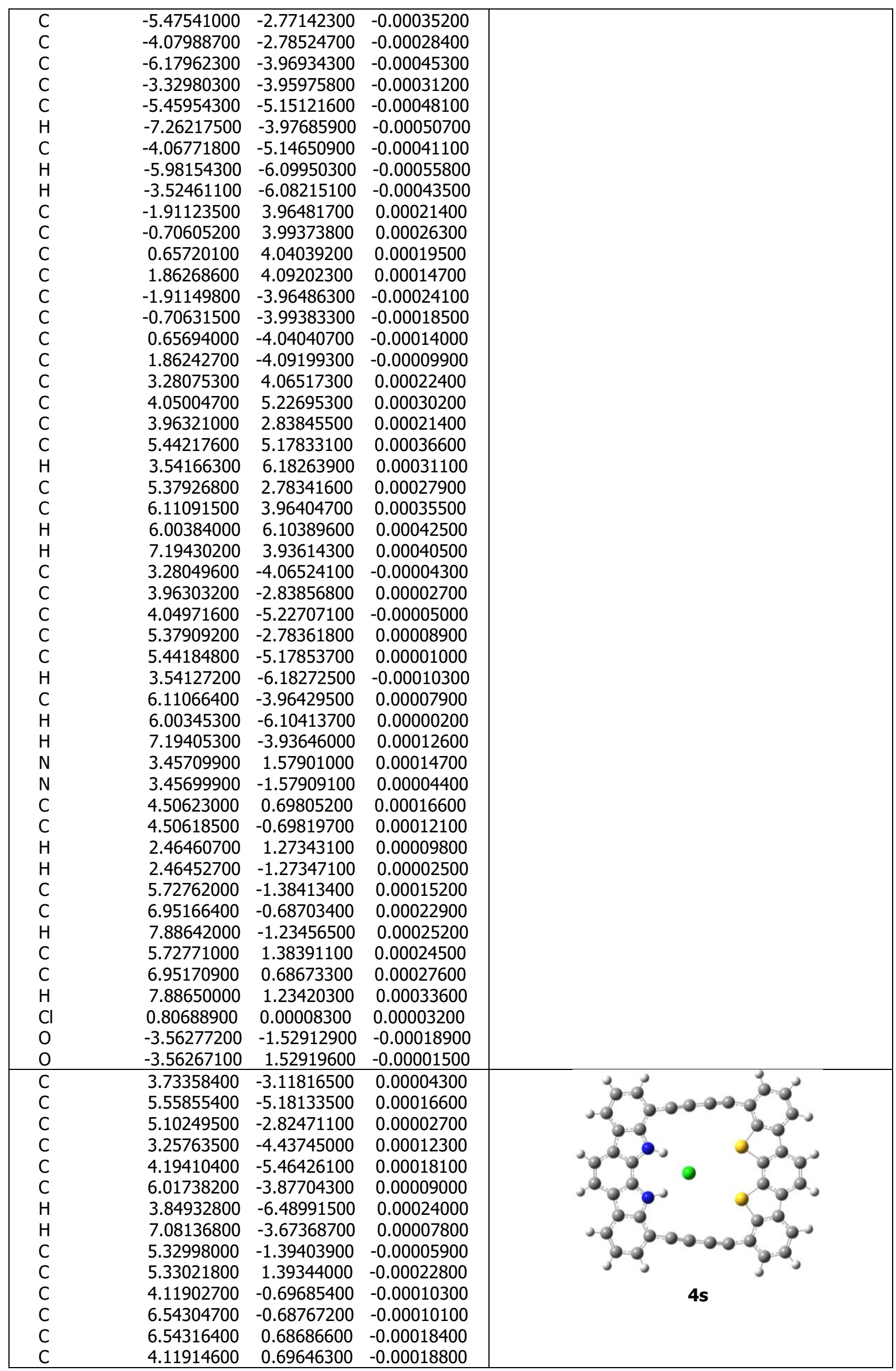




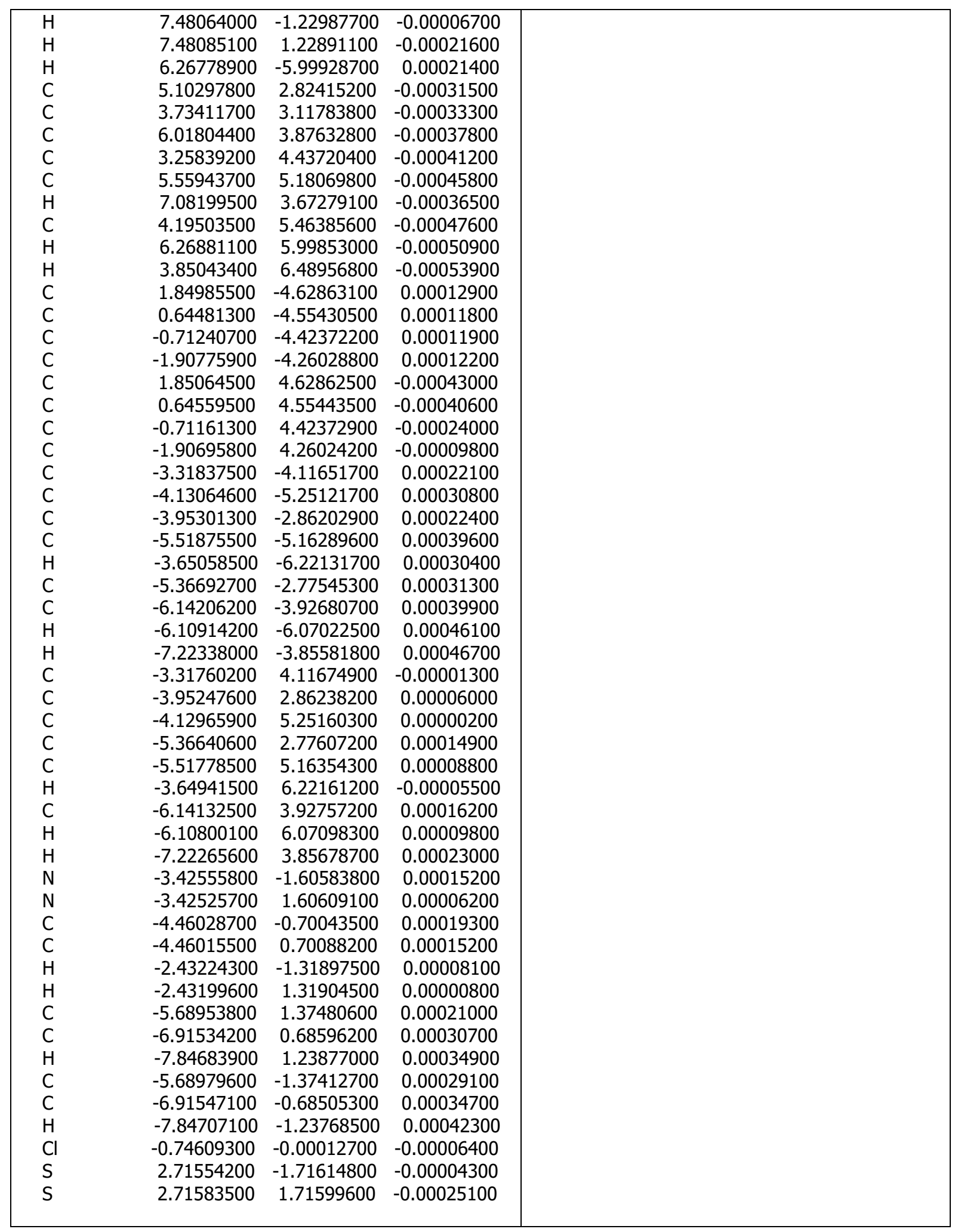




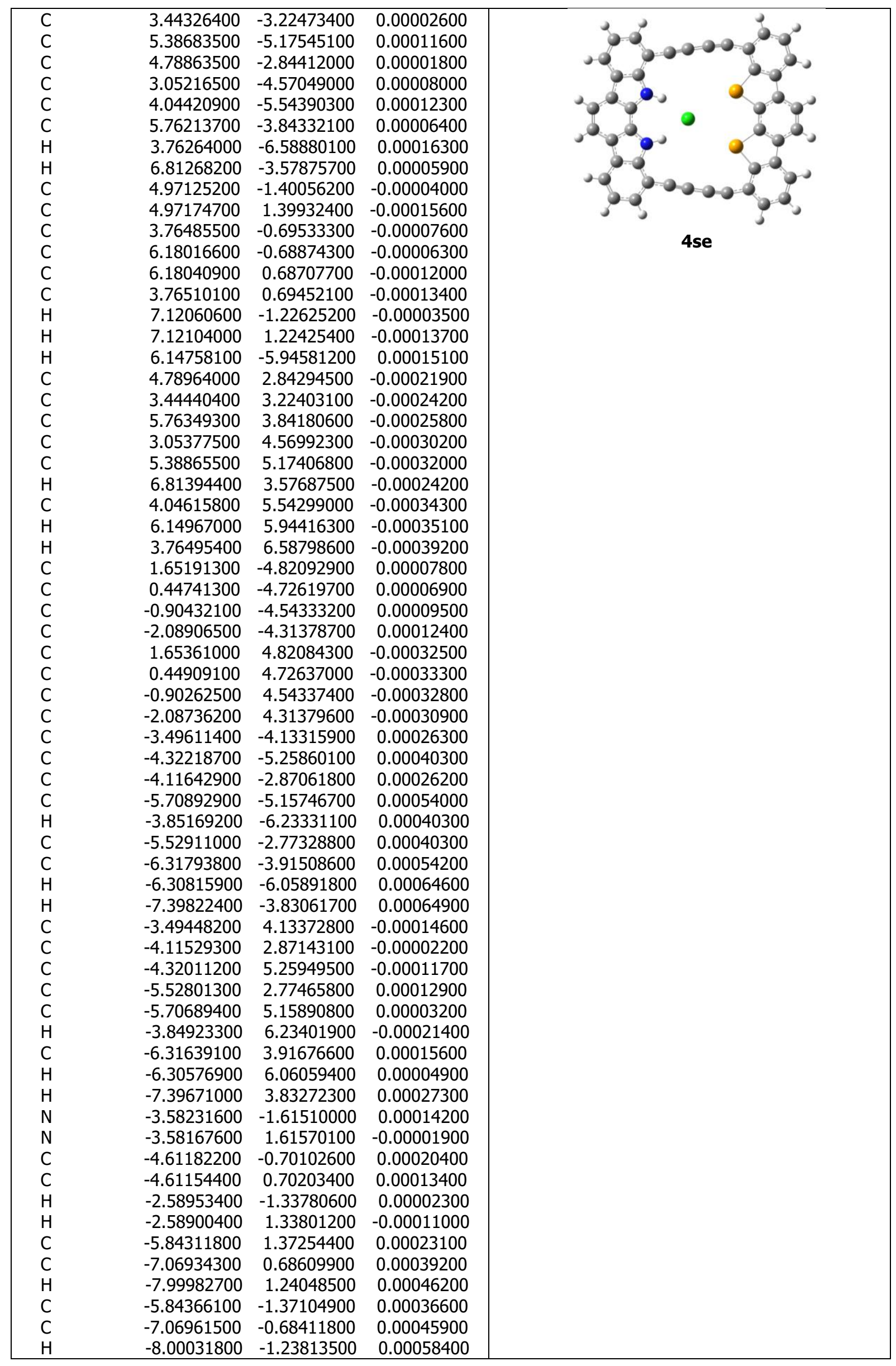




\begin{tabular}{|lrrr|l|}
\hline $\mathrm{Cl}$ & -0.88842100 & -0.00024200 & -0.00022600 & \\
$\mathrm{Se}$ & 2.23320000 & -1.79093500 & -0.00004000 & \\
$\mathrm{Se}$ & 2.23383200 & 1.79066200 & -0.00018700 & \\
\hline
\end{tabular}

Cartesian coordinates of the structures of host-guest complexes 2 in the continuum DSMO

model

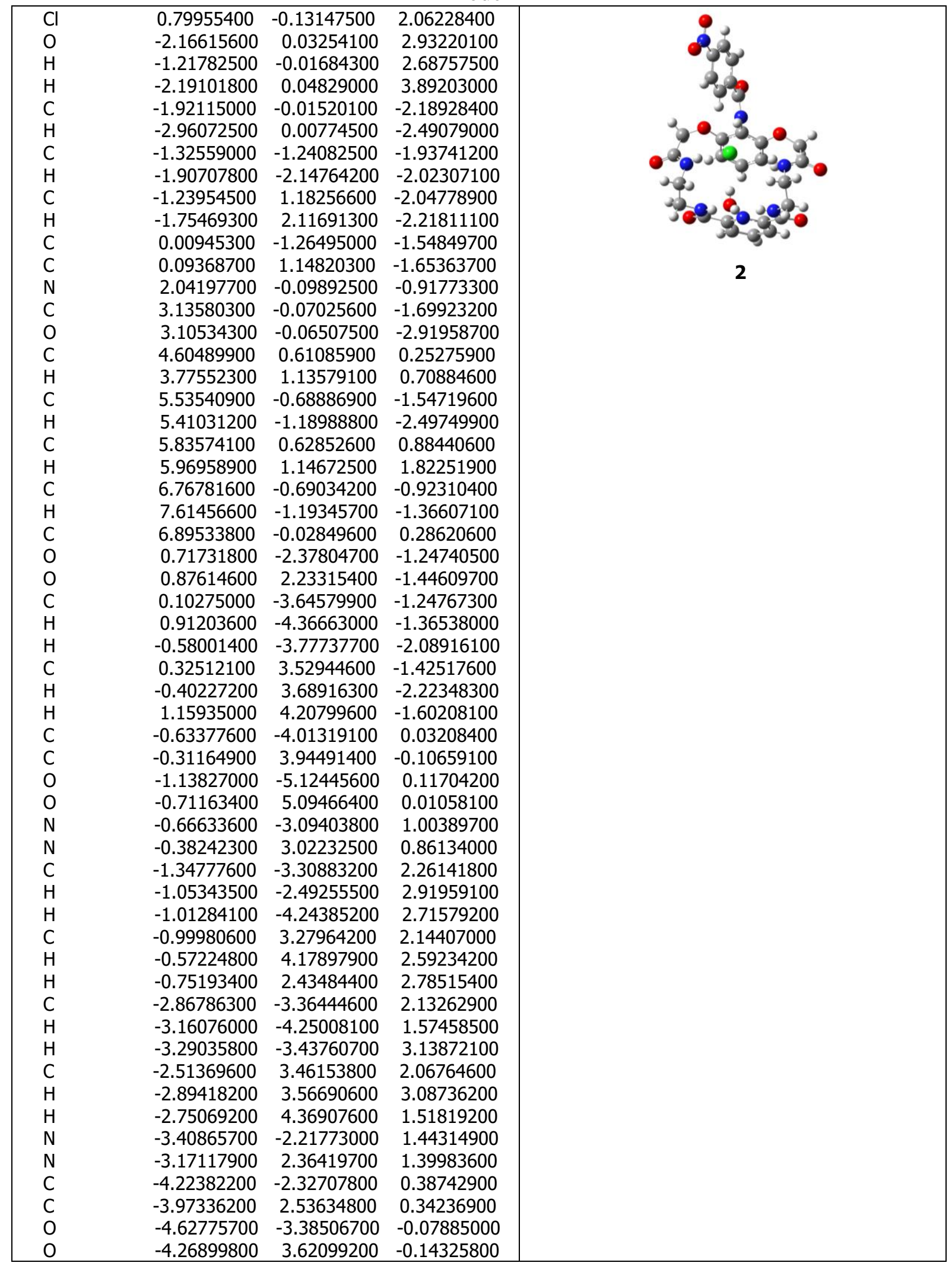




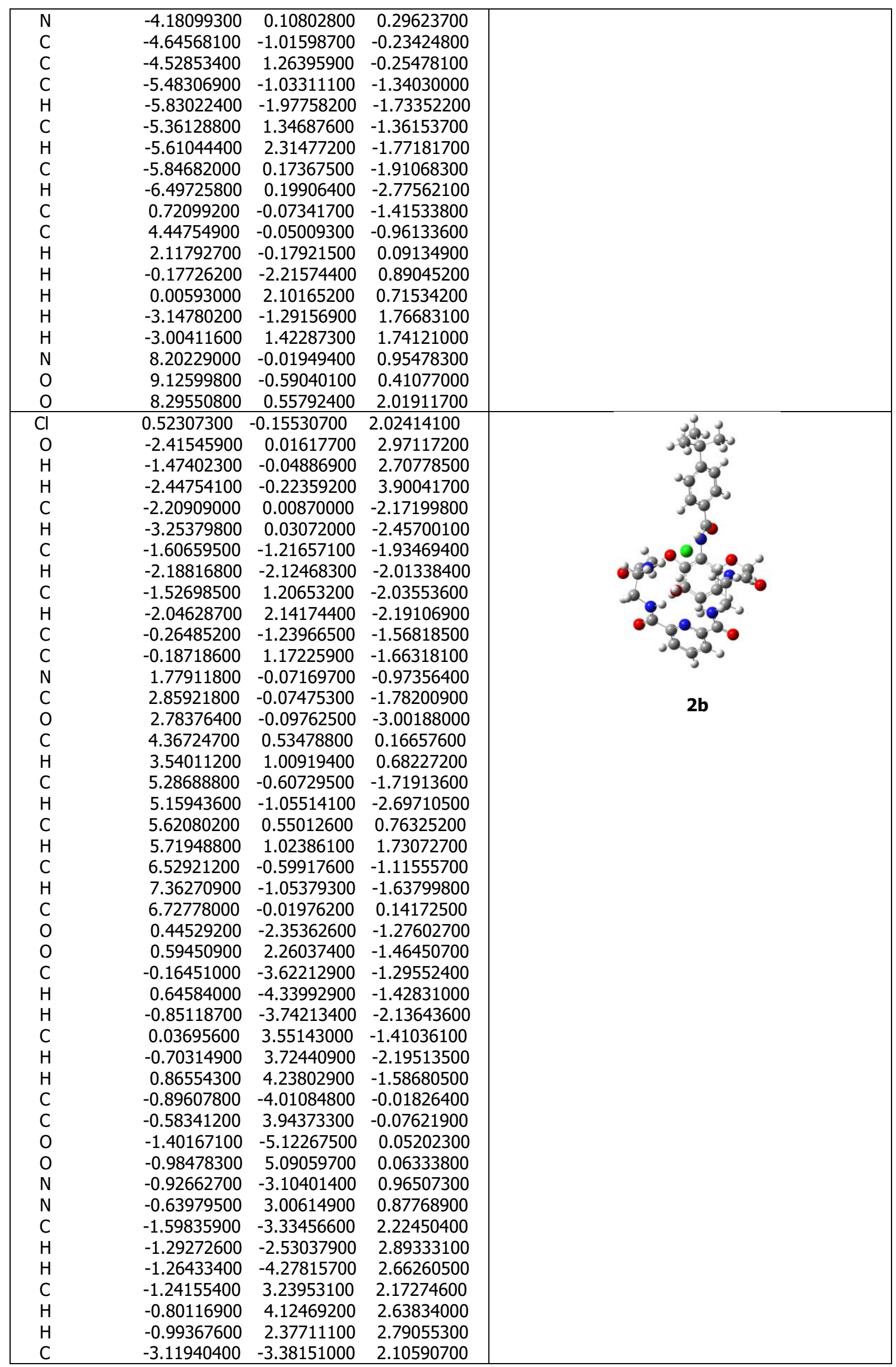




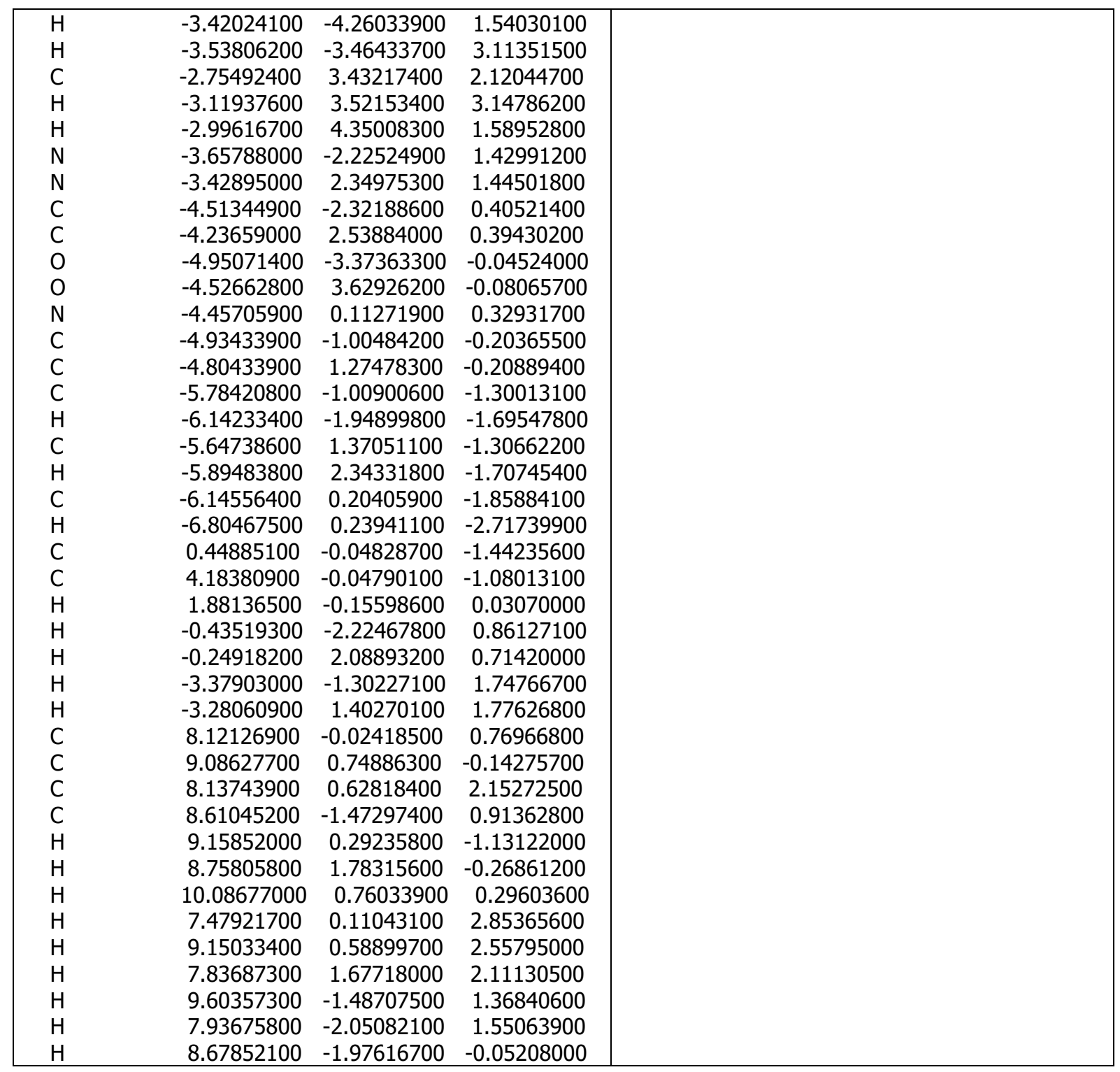




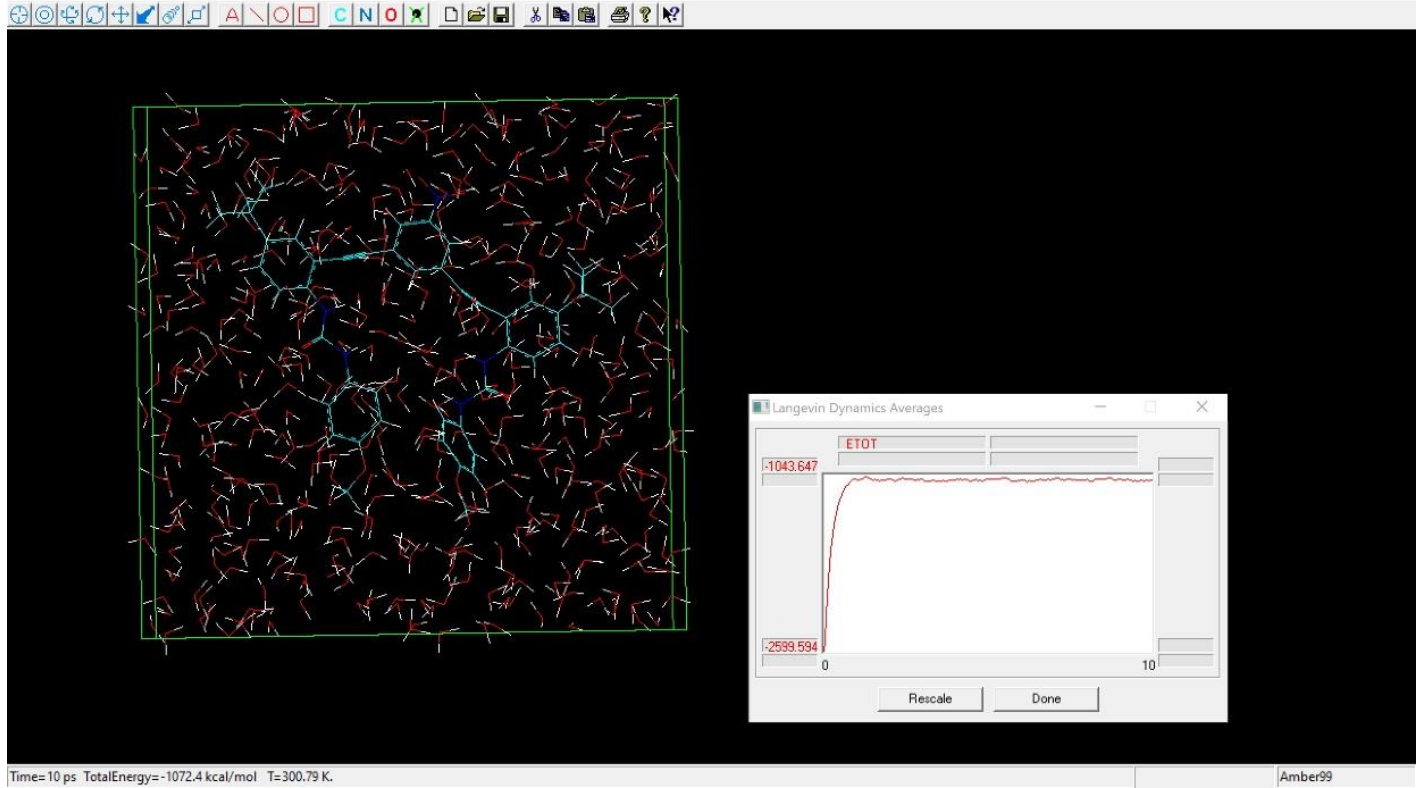

Figure S1. Illustration of the Langevin dynamics calculations

Cartesian coordinates of the QM/MM structures of host-guest complexes 1
H $0.3155934918-1.7026003591-1.5540053652$
C $6.6269169094-3.6548154787-1.0342701607$
C $5.7724863525-4.3839271296-1.8527035418$
C $4.4271699066-4.0818023329-1.9789778791$
C $3.8651566787-3.0196654775-1.2707506173$
C $4.7158816494-2.2784404108-0.4320934331$
C $6.062793287-2.6048712954-0.3328912885$
C $8.1193278138-3.9262834556-0.8896756599$
C $8.5426784627-5.1945464818-1.622370708$
C $8.8930368362-2.7328450207-1.4658797667$
C $8.4582649326-4.10521946270 .5939190596$
N $2.5392493642-2.6269812892-1.3571448393$
C $1.5348010956-3.2866844138-2.0457676035$
O $1.6847843092-4.3918171337-2.5372344517$
N $0.3861556917-2.5579251027-2.1094357339$
C $-0.7912171882-2.9572814559-2.7559276331$
C - $1.9531049379-2.2423780375-2.4596130067$
C -3.1509673611 -2.5804415002-3.0471587973
C - $3.2255677468-3.6448338234-3.9325421185$
C $-2.0762781245-4.3436953724-4.2536667732$
C - $0.8612689575-3.9994164345-3.674203516$
O $-4.4568090401-3.9096624933-4.4598507598$
C $-4.679398614-5.2007467593-4.9546355503$
C $4.2526956543-1.18286177270 .3415610614$
C $3.9701797539-0.2548046761 .0496734702$
C 3.82548977490 .81308866621 .9754892016
C 4.9629015751 .20048168722 .684260431
C 2.61565169261 .46725851412 .1824737026
C 2.53999863782 .49049869453 .1274553168
C 3.66616436852 .86530358883 .8568772652
C 4.8619921392 .22471625453 .5987235687 
C 1.32632726653 .20242823043 .3289426732 C 0.34202349663 .87126098393 .4961383232 C -0.79937817464 .69926000233 .6541171124$ C - 1.92721713044 .51304987072 .8412308643 C -2.99542302695.39729660193.007240983 C - 2.93430865356 .39635703633 .9538690084 C - 1.8350270226 .57859441324 .792908099 C -0.77119964645 .71707149044 .6113888435$ C -1.85718621397 .66724595685 .8614668833$ C -0.5418117247 .72968754036 .6387875294$ C - 2.11079793379 .03403161825 .2143487352 C - 2.99213696047 .37431725836 .850978798 $\mathrm{N}-1.91825643 .48923860091 .9042952018$ C -3.0436142253 2.9117052057 1.3361148584 O -4.17267545233 .25684710841 .6291595669$ N -2.763539426 1.92793753730 .4375550964 C $-3.80324583281 .2166299297-0.2010239284$ C $-3.8388827828-0.1687910398-0.107340493$ C $-4.8301965188-0.8885418968-0.7411555433$ C $-5.795223568-0.2352379131-1.5000783379$ C $-5.78517943421 .1516424762-1.5708348879$ C - $4.78799446171 .8681499658-0.9242006968$ O $-6.6681183934-1.0348277081-2.1785296708$ C -7.7540288358 -0.4200943295 -2.8235473665 N 6.07591389322 .72332496874 .2594201769 O 7.13136977992 .15671855564 .0644408327 O 5.967784413 .71273069344 .9496664331 H $6.1489776437-5.2231055078-2.4226250862$ H $3.8046691418-4.672964789-2.6266795345$ H $6.6707004464-1.99255454110 .3193589264$ H $9.6051719187-5.3833402269-1.45795124$ H $7.9878905834-6.0623056945-1.2599723555$ H $8.3831751412-5.1129508116-2.6960987197$ H $9.9688748328-2.8981492597-1.4036295603$ H $8.6264742825-2.5783079342-2.5129481394$ H $8.6597855271-1.8132445728-0.9283530962$ H $9.5107715231-4.37057184760 .7173816588$ H $8.2653439282-3.19940725771 .1666819535$ H $7.8481118748-4.90230757771 .0182945365$ H $2.2951269646-1.7664596232-0.88595063$ H - $1.9066872059-1.4100032943-1.7652721696$ H $-4.0505803213-2.0312038271-2.8179048662$ H - $2.1049714378-5.1647055286-4.9575280512$ H $0.0258980709-4.5540650308-3.9289807961$ H -5.7580521974 -5.3072712449 -5.0656408942 H $-4.2049258856-5.3703665157-5.9285314729$ H $-4.3185259752-5.9681252891-4.2611745136$ H 5.8963956140 .69244794142 .5106731845 H 1.74054540381 .16907660011 .6094172591 H 3.60930236513 .65950935234 .586065417 H -3.8774657021 5.27935516232.4016050632 H -3.7853832464 7.0605939061 4.0419492119 H 0.1198745065 .79753643035 .2176301402 H - 0.60031482328 .51483222637 .3944188432 H - 0.34069820576 .79180945727 .1588555581 H 0.30512804357 .95320905085 .9891168085 H -2.1101182657 9.81475974585.9788495913 H - 1.34298816359 .26604403384 .4792314194 H -3.0796247062 9.067771893 4.7131086605 H -3.0096017178 8.1274669047 7.6421687819 H -3.9654219105 7.3831289896 6.3580014222 H -2.8623848515 6.3951908764 7.3090905835 H - 1.03157597773 .03834151141 .7386683229 H - 1.83169479711 .51753062770 .3620904449 
H -3.0695472659 -0.6755565252 0.4606475477 H - $4.8544809426-1.9664450669-0.6669879974$ H -6.5320045849 $1.6896361934-2.1383178775$ H -4.781571063 $2.9475308677-0.9864621115$ H -8.3767301368 -1.2199813599 -3.2217460192 H -8.3518874216 $0.182763493-2.1284501885$ H -7.4316585348 $0.2211603454-3.6511773489$ Cl $0.05767509170 .1049453245-0.0292036342$ O $1.2949637813-1.7048754372 .5603424739$ H $1.1024735152-1.36591057691 .6759101805$ H $0.4326476753-2.07848461032 .8375106251$ O $-2.81675458692 .9787127436-4.2332605915$ H - $2.57473793082 .0305059493-4.2225685413$ H - $2.40038312783 .3155254295-3.4219740504$ O $1.8508270251 .8830514922-2.1638089274$ H $1.34281365461 .3867687969-1.5013425297$ H $1.30767801641 .7605279519-2.9698192648$ O $-0.30810168155 .6587825306-0.0539140357$ H - $0.73632143635 .1255202952-0.7506048495$ H $0.63190329395 .5197545285-0.210134712$ O $3.59739851843 .7619669195-1.4146262599$ H $3.09147791944 .4584951134-0.9690440681$ H $2.90979035963 .0875337836-1.6206102051$ O -3.2506495092 -2.9898927057 1.7403105309 H -3.2154370416 -3.8415600717 2.2195166417 H -3.7859887721 -2.4223203445 2.3422634687 O $-2.07987233150 .3674221633-4.3295389431$ H -3.0429192106 $0.1726958872-4.2799372186$ H - $1.7792654399-0.3362505679-4.9497580297$ O -4.5629702959 -1.5257723451 3.479536992 H -4.7481546983 -0.5718932799 3.2537197046 H -4.032855009 -1.4499404972 4.2992495682 O -2.0646278875 1.07347020444 .2132887146 H -1.10218630060 .89945199994 .2715092943$ H - 2.44209116230 .20011422174 .3636286463 O $0.3338993611 .5302694582-4.4270485239$ H - $0.49011393921 .038469698-4.2036436743$ H $0.02560876112 .0383694694-5.2130359854$ O 0.50510016430 .2397177464 .4777318493 H 1.23807996530 .06369125285 .1056515292 H $0.7710313898-0.2901323053 .7117399157$ O $6.6427991094-0.3730288988-2.6105204567$ H $5.7266101751-0.0516820333-2.6970703556$ H $6.8778378726-0.1061529926-1.7046585895$ O $3.6268064471-2.74680096663 .3904916279$ H $2.7909805113-2.30497915243 .1178392939$ H $3.7116911816-3.44074472812 .7058373167$ O 2.5785212475 .97239969880 .2196181052 H 3.41747995345 .67212880130 .6176201231 H 2.08443065586 .24741785391 .0177708336 O -5.19421468070.91293729572.8740060169 H -4.82722899371 .65214607712 .3671858698$ H -6.148407888 1.15434384022 .9309151977 O $-2.6189419146-5.2634044729-1.2025037487$ H - 2.4464306856 -4.3198855638-1.3012170238 H - 2.1413565006 -5.4668624599-0.3505788674 O 4.54365132064 .8405660671 .7556185857 H 4.03692049294.27365031912.3488876884 H 5.0643101654 .19505813481 .2359739921 O - $1.1383999345-2.44568610763 .4221006665$ H - $1.7349409294-2.01304967064 .0637892518$ H - $1.7348171245-2.63506459022 .6727683795$ O $-1.3355191458-5.84821002450 .9576766562$ H - $1.4949432334-6.81103374461 .1010408238$ 
H -0.3899042661 -5.7483287819 1.2570510776 O $1.0744149534-5.67218463771 .9285043684$ H $1.976185125-5.34379596651 .7592538026$ H $1.1073003176-5.87853550022 .883950331$ O -5.0993504805 4.0269066537-5.163870798 H -4.2928966878 3.5634615293 -4.8431920034 H -5.2371029638 3.6194562419 -6.0453532698 O $-5.6877615662-4.12180205711 .1359117041$ H -4.8354823854 -3.6580687718 1.1269857347 H -6.3191098312 -3.4270106435 0.8693965553 O $0.6812925146-2.9780595998-6.9350806554$ H $1.1358212846-2.7120817603-7.7520035663$ H $1.4281264215-3.0465778533-6.3014535472$ O 5.8199791643 .13361409580 .0904213025 H $5.13612317653 .3239805147-0.5812597772$ H $6.59929526363 .6363164682-0.2253082962$ O $-1.60834546394 .540509081-2.2094781948$ H - $2.47934306214 .9633367645-2.0825430756$ H - $1.24882473764 .9952670854-2.9978936064$ O 1.63936617876 .74627649612 .6758059834 H 2.07456805177 .06654152483 .492514161 H 0.86107967176 .28791155543 .0148876003 O -0.10105783372 .20766117756 .6451546526$ H 0.43551790322 .08717396515 .8497642529 H 0.44847150882 .82535934417 .1936699931 O $2.5895080148-0.30076223956 .0566544607$ H $3.4687195653-0.40933753795 .6269149902$ H 2.809643320 .23201348766 .8501334995 O -6.4770006177 $4.783709768-2.9500967075$ H - $7.31415370064 .3102718577-2.8058840572$ H -6.1239773149 4.3724619821 -3.7654494987 O $-2.1490071892-7.3912275608-2.8326650047$ H $-1.2430853224-7.7045430307-2.5966698537$ H - $2.2060303123-6.5298546688-2.3630800263$ O - $2.6878147486-1.47095900825 .4687141807$ H $-2.3348534028-0.77937311276 .077994229$ H - $2.6657618544-2.25197755326 .0507682785$ O $-2.9559863917-5.33728990133 .0190978189$ H - $2.429349817-5.50181986052 .2055847274$ H - $2.4421472034-5.84978051023 .6861635532$ O $-1.3902623266-1.4498370573-6.1468933488$ H - $0.638440262-2.041413811-6.3727335788$ H - $2.1686001681-2.0026213571-6.3803375075$ O $-4.7186254475-0.0931025537-4.4826816909$ H -5.2514407388 $0.1524293052-5.2629667545$ H -5.3783671845 - $0.4394314138-3.8709891887$ O $4.3125739749-4.0240972385 .6427747019$ H $3.9773960186-3.47914964214 .8940898118$ H $5.0272518626-3.46761894066 .0082614703$ O $2.3085632594-0.4960123216-4.6903683231$ H 2.5114477919 -0.4996375694 -5.6567079209 H $1.54627080340 .1075677167-4.6379974655$ O $4.16818705250 .6690079619-3.1732562253$ H $3.54361340071 .1757093078-2.6290321303$ H $3.55409794590 .1082450369-3.6988345651$ O -9.600986939-1.1549789773 3.8446822091 H $-9.4080733173-0.28556820784 .2448389104$ H -8.6970357332 -1.5294442207 3.7289811947 O $2.8164230802-3.1774555431-5.3114128007$ H $2.6637635528-2.3496161278-4.8203943578$ H $3.7982160437-3.2076477524-5.3727993157$ O -7.1514945195 -2.1212762629 3.6123686836 H -7.2041532385 -3.1085471627 3.6588453799 H -6.184951696 - 1.96308719363 .503972957 
O -1.94962451477 .65134297440 .7338291282$ H - 1.42793551088 .24918467181 .3034252229 H - 1.31233900476 .93324887630 .525591303 O $5.2886631779-5.83349900153 .873053816$ H $4.9192429417-5.27370172864 .5817765292$ H 6.2198657855 -5.5187901573 3.830651127 O $7.22521442810 .8072795509-0.1678301339$ H 7.68017817630 .66957338220 .6851937293 H 6.60353469611 .53319893820 .0386961742 O 1.86624702619 .20683079951 .4582894793 H 2.82839088899 .44109455271 .4625169766 H 1.86435339578 .31195120021 .843664677 O -4.1010840236 5.7235252372 -2.0516879649 H -4.980418956 5.3103213514-2.1761681336 H -3.9874898943 6.1868738388 -2.9043324152 O - 2.45048521733 .46146546017 .1684320525 H -3.0194789602 3.3478607336 .3775979373 H -1.6045183759 3.07307970526 .8695062502 O $7.8082205591-5.03256430853 .9686352313$ H $8.5287011611-4.39118391323 .7989330976$ H $7.9633679861-5.2981264594 .8966557317$ O -0.66577885169 .75339842551 .9428836423$ H - 0.72914217510 .71839556561 .7667720213 H 0.27680640719 .56680221441 .7323526324 O -8.5606891421 0.37522712880 .560705134 H -8.1294853477 -0.49414669050 .4773202094$ H -8.1190452998 0.76305673281 .3334629074 O $-3.5655131289-2.8649913026-6.8565460543$ H -4.3858316785 -2.4319278881 -7.1891573828 H -3.8876174778 -3.2856543906 -6.0357855921 O $-6.0736963344-2.08053890326 .2247896958$ H -6.1211913382 -1.1966371579 6.6402247282 H -6.585438177 -1.963722474 5.4101514236 O 4.38419660089 .91131043131 .3355246181 H 4.515722797110 .88283791481 .3789133256 H 5.04614092029 .6344015870 .6650867621 O 1.17657066743 .9131395228 .1908346664 H 1.99285629063 .97453870318 .7168268928 H 0.57618887814 .51170224358 .6725340314 O -3.84468452072 .94799845694 .8953770132$ H -3.2399738558 2.2285745667 4.5924071932 H -3.960473945 3.4655571964 .0888557734 O $3.7524326503-4.98762335531 .802316981$ H $3.963147932-5.63286787481 .0952302439$ H $4.2876178763-5.33343316472 .5423797312$ O $-3.8311032369-8.2514004363-0.8583728536$ H -3.094877498 -8.1073223271-1.477467825 H $-4.3962413478-7.4749607193-1.0352838473$ O -6.3615570590 .34675466737 .3867944187$ H -5.4505384001 0.37920793147 .7737150222 H -6.3869160783 1.2175000883 6.9117005749 O $4.9478455484-0.94275328624 .9856655116$ H $4.6357222302-1.52026904224 .2651041575$ H $5.6197828298-1.48024747535 .4340268459$ O $4.11703618654 .4447894342-3.9895209204$ H $4.09969458024 .1158201629-3.0682123595$ H $3.16252531464 .3752612199-4.2339132999$ O - $2.0709702179-8.35229406331 .3196737089$ H -2.4093850152 -8.7060854684 2.1711050795 H - $2.8153894376-8.54895085560 .7117858978$ O -7.75077102421 .56561138182 .9960991248$ H -8.30761135042.27937329492.6083201019 H -8.1692406063 1.44485639463 .8709363856 O $-0.83806355613 .1293998672-6.2456282813$ 
H -1.6429376133 $3.3144049456-5.7369531524$ H - $1.0072593853 .580151962-7.0960122587$ O $-4.75185320899 .7332349487-2.4658541669$ H -3.9809013497 9.7992118729 -1.8651923406 H -5.3968927494 9.2411553708 -1.9128562507 O $0.2212646727-8.4199382793-2.1503745421$ H $0.2988220835-8.5926669847-1.1827152115$ H $0.2730042958-9.3239981436-2.5133898782$ O $0.1470031462-3.66120701755 .4661110812$ H - $0.2589019448-3.25920088054 .6685763103$ H $-0.583797129-3.61161161876 .1200094628$ O $-8.61451070028 .8243515447-4.0451823733$ H -8.7411948917 8.6930292886 -3.0812893566 H -8.38867175 9.7782415444 -4.092259844 O $-1.39102497670 .4709559141-8.0164920169$ H -0.4915161502 $0.8719127551-7.9409812395$ H - $-1.3988407802-0.1459147639-7.2537459276$ O - $2.7937668684-0.9919161774-9.7740682605$ H - $2.2202458053-0.4950742137-9.1529553493$ H - $2.6529053499-1.9174617215-9.5107821707$ O $-4.05177592566 .5061882731-4.6574769113$ H -4.5668282911 $5.7041021526-4.8868752573$ H -3.2907215212 $6.437920306-5.2758475156$ O -6.6140009284 -3.8125481747-1.8955766197 H $-6.5584397322-2.8514632383-2.0229300299$ H -7.5730872451 -3.9566703244-1.8216332662 O $-5.26568184593 .0001262085-7.6688095578$ H -4.4824770651 2.6771445386 -8.1710589124 H -5.9165466214 3.1828249978 -8.3873779441 O $-7.25378885756 .7572745241-4.9492947471$ H -6.8518486919 6.4293086214 -4.1290094326 H -7.7460304625 7.5592438858 -4.6429574022 O $-5.1642010748-6.0007452261-1.666049581$ H -4.2495539587 -5.6491888917-1.5714288799 H -5.6770782499 -5.1588950295-1.7972019015 O $8.81272169492 .0426437264-2.0017356024$ H $9.51834123151 .3676743539-2.1278861415$ H $8.21166562791 .5700487612-1.3913770061$ O $2.6063870156-8.78574593761 .6472663893$ H $3.2068434151-8.13120597231 .2397781853$ H $1.811468812-8.72953706121 .0765789542$ O $-0.53434541675 .727501133-4.4227779352$ H - $0.19020034476 .6244058065-4.202442573$ H -1.0856203597 5.909901606 -5.2190556146 O -1.38120758432 .26623049719 .7343664402$ $\mathrm{H}-1.88938702391 .549284143410 .1921026915$ H - 1.95798470522 .50539904598 .990866885 O -6.16817361022 .71458645876 .2512752535$ H -5.3528542453 2.8057207432 5.7069408981 H -6.8238450582 3.24157261665 .7449568339 O $1.0727730889-6.00877169484 .621090378$ H $0.8032099566-5.1259044484 .9586873448$ H $1.8347964374-6.22402021525 .196745416$ O $-5.1090003506-5.54972158524 .5617443896$ H -5.9103665321 -5.1664534579 4.1339189914 H -4.3997316133 -5.3931543952 3.8930425942 O 6.42432639126 .34685758083 .0634421473 H 6.01081162667 .17445513693 .3849077215 H 5.65737890475 .84639289092 .7258690329 O -9.45726833413 .36736357112 .0699242086$ H - 9.52947402314 .2070644532 .5686603263 H - 10.20584372733 .42249222661 .4578777705 O $2.0834072669-2.067257367-9.2654740929$ H $1.1882331375-2.2573468409-9.6233493446$ 
H 2.6261669369 -2.1367661695 -10.0699869095 O $6.5427029827-2.63669377376 .5121882844$ H $6.6649595981-3.35933087237 .1723211194$ H $7.4683279295-2.49181932686 .1915514912$ O $5.77729449591 .3210807415-5.2839352782$ H $6.53158772251 .8351216786-4.927240636$ H $5.2015815351 .211977189-4.4986964677$ O $1.6910023092-2.49774427937 .3091773889$ H $2.0807504169-1.73170264976 .8317391898$ H $1.2197215046-2.96330931676 .5813890056$ O $-0.0977957372-5.6319860865-6.8192088618$ H - $0.0866937337-4.6639216466-6.9341059967$ H $0.8498708433-5.8167974765-6.666232174$ O -1.63928779270 .16511970127 .2732932886$ H - $1.0628309934-0.32971963957 .8917608325$ H - 1.05460887310 .9208073957 .0042440245 O - 9.19001707361 .10807861045 .2704330686 H - 10.0199413471 .575849785 .4629331687 H -9.05635341590.5652484298 6.0738256604 O $3.1897285629-6.31355656216 .2699336058$ H $3.5836265854-5.42073767876 .1080525873$ H $3.446973592-6.47980118767 .1958306829$ O $10.2846347451-1.288486051-4.581191151$ H $9.8445663553-2.1532088311-4.7455070123$ H $10.1949196037-0.8364332833-5.4376643049$ O $0.2520206968-9.13170644480 .4072193265$ H - $0.6037394207-8.81049473510 .7877037491$ H $0.2372851569-10.07304942560 .6550975145$ O $6.9259356263-1.1642851957-5.1810784446$ H $6.5223955173-0.3059653626-5.4189630305$ H $7.0355840638-1.0501972362-4.2110835312$ O $9.8609320243-3.28034140493 .5125544304$ H 10.744925856 -3.668700662 3.6586755259 H $10.0141432124-2.75136680692 .7028590126$ O $1.56312107734 .1085963255-4.5951270642$ H $0.78531768474 .7199781258-4.5525906207$ H $1.15046266333 .2538614597-4.3868669098$ O $2.8962576241-0.4867707066-7.2629040229$ H 2.7087749548 - $1.1135175782-7.9999252955$ H $3.7459492195-0.0727398392-7.5458398351$ O $2.16702652757 .911746968-1.6964094724$ H $2.25663288037 .205887456-1.0233783766$ H $3.10833784938 .1397330953-1.8778163052$ O 0.88590890370 .96121836649 .8336999425 H 0.07834818671 .52804857299 .7443641836 H 1.33112778171 .385322998210 .5965902206 O $2.3491083301-7.1467191671-3.2296478313$ H $1.5435459019-7.5601826389-2.8529752408$ H $2.1909317541-6.2031821612-3.0704071947$ O - - 7.5070751792 -4.7064874338 3.7665508339 H -8.2232636681 -4.82356381084 .4279433185$ H -7.8723700654 -5.2160625713 3.0029695084 O $-4.23035378075 .600878473-8.0710787308$ H $-5.00379858486 .1877750723-7.9000565588$ H -4.5957702929 4.7217040295 -7.8859693041 O $-1.03333070310 .0705740672-2.7850805722$ H $-1.623486967510 .546927793-3.3949944562$ H - $1.571612117110 .0319983893-1.9647111176$ O -4.47082929256 .84145273470 .4041585339$ H -3.5544788058 7.1245157226 0.6213920272 H - $4.32339013336 .2943104247-0.3946303941$ O - $2.72645978199 .8346312801-0.6770889624$ H - 2.972818328410 .36971573080 .0984865564 H - $2.46282905848 .989881282-0.2576160266$ 
O $-4.3282679738-8.707139492-3.8965642728$ H -3.5434169725 -8.2298309298 -3.5586571802 H -4.6715741583 -9.158833717 -3.1099410276 O $-4.21038865859 .1644973862-4.9608018966$ H -4.16439463 8.1838247631 -4.8756008878 H -4.499993154 9.4165203434 -4.0465625164 O -0.34516026295 .70025957279 .7007345451$ H - 1.27363569765 .40393781479 .6031459299 H -0.31997896236 .026816782910 .6116302222$ O -8.8448089609 -0.6067162415 7.3496117575 H -8.7970027286 - 1.58963596237 .3049580968 H -7.899824519 -0.3481478427 7.4417929181 O -3.9744297647 0.3732914859 8.4536239195 H -3.1116772949 0.36943330777 .9691553915 H -4.0439914292 -0.5765873395 8.7179475036 O -7.651151388 -2.2068966274 0.9013989237 H -7.5479521064 -2.0512019445 1.8549731606 H -8.4453580992 -2.7878814891 0.8626537324 O $-6.1708671222-6.68388823210 .7770936742$ H -5.9376293532 -5.7620340227 1.0454639214 H -5.9656741751 -6.6475695985 -0.1745367519 O 3.11553735960 .85508128268 .4101894096 H 3.51739215860 .21730609429 .0351002089 H 2.24265423771 .02066985628 .8326780962 O $-0.8447978232-7.8266048429-5.2909820061$ H - $1.4330994572-7.6135590422-4.5514957238$ H - $0.716585066-6.9734491511-5.7379077985$ O $8.12307465464 .3484276638-0.6772871864$ H $8.56284507683 .5875182389-1.1062210255$ H $8.27711282225 .0705041227-1.3195206654$ O $6.40357280158 .9936062427-0.2314061001$ H 6.79597872898 .1145716460 .00747391 H 7.00070640559 .60479448950 .2435576916 O -2.4284490962 -3.6617747916 -9.1108118734 H - 2.7880086965 -3.4825342626 -8.2117986189 H -3.100735575 -4.273958447 -9.4771263561 O -4.39944563142 .58801340399 .8204807862$ H -4.7140025618 2.2867281713 10.6930005541 H -4.2538146681 1.73228922079.3414080728 O $-1.4981289935-6.82941318564 .6768177672$ H $-0.538223039-6.66939418694 .5395462794$ H - $1.5402432869-6.91479087575 .6549112945$ O $-8.7543109398 .2137438004-1.4438091038$ H -7.7940335962 8.238056056-1.2194113497 H -9.1310765094 8.7341992504 -0.698362402 O $5.4667531614-3.3715137786-5.6049454659$ H $6.0583168434-4.0871338882-5.2919400975$ H $5.996300601-2.5658858841-5.4142616262$ O $-1.8402794248-11.2607975283-0.820128334$ H - $2.427832613-11.6241358527-1.5149676786$ H $-2.5047439348-10.9802589488-0.1384543043$ O $0.48775164088 .073835314-3.7390368946$ H $-0.13377050268 .7670866487-3.4214067767$ H $1.07722988627 .9585315213-2.9550784195$ O 5.19052394768 .71899911113 .5830836668 H 5.91108046019 .32235802023 .8679283143 H 4.89287710199.14202165812.7492966569 O $-6.32255548387 .1174930573-7.4310271373$ H -6.6578391826 $6.9676752296-6.5179084136$ H $-6.17384784778 .0848862018-7.4375381127$ O $10.60215499050 .079008819-2.3368407041$ H $10.4105682304-0.4761640952-3.1320089227$ H $11.1720495695-0.5019397074-1.8039175821$ O 1.116818118110 .21127906274 .2937709611 
H 1.353418954410 .92450189553 .6720255431 H 0.31547620849 .84215438083 .8964528329 O 4.745145346212 .60649701691 .5526339015 H 4.036640605813 .15570194661 .1689931063 H 5.458506870912 .6943609630 .8926431047 O $5.2273344536-8.49009590264 .1926597644$ H $5.1524483343-7.51143314564 .1291666507$ H $4.451919806-8.72496151614 .7365349766$ O $10.7266769575-1.789829731 .3716844487$ H $11.1806938628-2.40964327640 .7811787513$ H $10.8032569925-0.94548252730 .9096164557$ O -5.5082834605 -9.0174245664 1.958009681 H -5.702610884 -8.1620596943 1.5121731935 H -5.6249105314 -8.7752139445 2.9057220622 O $5.67382007326 .5119889493-3.4168766794$ H $5.07820600145 .7619782124-3.6503656903$ H $6.07128370576 .7425147339-4.2821230655$ O -3.6647719055 -10.7219147582 0.9880274448 H -3.7553421528 -11.5672875539 1.4596625182 H -4.3389103588 -10.1495563572 1.4201695665 O -13.8346198875 -1.4944391017 5.8004222283 H -12.8794397666 -1.6801308947 5.8945265269 H -13.8425917364 -0.6741463168 5.2844885302 O $-1.6492859781-3.22142403657 .4251791541$ H -1.9443121735 -3.85630383 8.1072803691 H -1.2454753441 -2.521318849 7.9648153979 O - $0.2194595262-2.9127155107-10.2962930279$ H - $1.0256184995-3.2408954417-9.8252497268$ H $0.1706382126-3.7419951214-10.6352764519$ O $2.6178467733-5.6295784629-6.4648249504$ H 2.6546241665 -4.7752538566 -5.9881285019 H $3.4851990755-6.0241917777-6.2374495683$ O $6.7915771332-4.57584614918 .3164747924$ H $7.2265236312-5.20247559377 .6971309894$ H $7.1859295756-4.83000004969 .175632663$ O -10.241993614 6.191743872 -2.2469648664 H $-9.63608479256 .9318595912-2.003844904$ H -10.6020125551 6.504993167 -3.1090836822 O $7.9529348664-6.20441076016 .4823393838$ H $8.7188300225-6.69394648216 .1381485891$ H $7.3623193913-6.92941966476 .7979444184$ O -0.926225613812 .38993076541 .6080502571$ $\mathrm{H}-1.227962887112 .75696418390 .737044878$ H - 1.625385926312 .71295024762 .2145265134 O 2.20706468656 .91830181099 .7015244459 H 1.29805074946 .65460437429 .4779612784 H 2.28452439697 .80322566389 .3056940578 O 7.41051639436 .72291838090 .5948937871 H 7.15561393696 .55579464731 .524376533 H 7.7145633075 .85046808850 .2894545407 O -3.0309324428 $2.3083377075-9.004158274$ H - $2.4226498873 .0806553504-8.9413311328$ H -2.480242337 $1.5981168586-8.5946407828$ O $1.757294454110 .49701356-0.9044003716$ H 1.668920950710 .22516978220 .0293739345 H $1.78306178849 .6249925673-1.3444790426$ O 8.48993608970 .68271145612 .2817727391 H 8.0099207731 .23900369952 .9328034458 H $8.727541475-0.09179966282 .8034896025$ O $10.44847874770 .147929696-7.0076364797$ H 9.98197359340 .5928179625 -7.7373541081 H $10.7436290599-0.6895227221-7.4204058924$ O $-11.1852522501-1.97911798015 .9458918325$ H -10.6790371166 -1.7670011395 5.1403595405 
H -10.550265633 -2.5015633962 6.4606658353 O $4.6697065229-3.48547893229 .667263986$ H $3.843348086-3.94642254229 .3821475572$ H 5.3520610835 -3.9164617871 9.1102578314 O 2.86278803438 .08132443274 .7087168233 H 2.3129833484 8.888524328 4.6700972379 H 3.71052082778 .36936646894 .3000389766 O 8.40776820454 .48238504673 .2178320046 H 7.80408958585 .2499625213 .2937437208 H 7.82587752073 .75516467283 .5033830536 O $4.4215812776-7.16630599510 .3893592429$ H $5.191976355-7.59669732840 .8148737598$ H $4.3961071047-7.6094962627-0.4862259469$ O $-4.59236699930 .7544019818-10.645134077$ H -4.1599324844 $1.4615699708-10.1315545838$ H -4.0499472915 -0.0263042387-10.384113766 O $7.0915073826-0.3651604887-8.9846468827$ H $6.6475125321-0.9369737187-9.6519767314$ H $6.32005018450 .0857192701-8.5766183224$ O $5.05076588360 .941367503-7.8678320993$ H $5.05509851861 .7489658947-8.4076855746$ H $5.33290121691 .2566669221-6.9787036326$ O - 11.12237098996 .3005659340 .2722326652 H - $10.85909094716 .2539274016-0.6745872063$ H - 10.3154146746 .59250087020 .7088548294 O $-0.1126513743-1.34201121178 .941276056$ H $0.6364268158-1.76863399678 .4664278992$ H $0.3241027784-0.55777538069 .3594006066$ O - 1.61784756663 .52655485212 .1610609814 H -1.54875988233 .191248384811 .2439028683$ H - 2.52352642353 .868572479112 .2039176129 O $-6.31355307660 .5876856113-6.575857895$ H -7.2865637765 $0.635760581-6.4946176972$ H -6.0725712624 1.4595206313 -6.9374291641 O - $-11.8417658857-0.80636813032 .4741177345$ H -10.9765589636 -0.96351787942 .9184947769$ H - $11.5929191403-0.22443222581 .7252780194$ O -13.19388969830 .73213841034 .1760503783$ H - 13.51370193741 .41541880963 .5608433438 H - 12.76977584960 .0897960423 .5627401601 O $-5.0510393911-4.39920713896 .9325528939$ H $-5.0084019078-4.83933783476 .0479037156$ H -5.3910427854 -3.5031600493 6.6807592465 O $6.2874066551-8.02128964617 .5273649721$ H $5.4951857673-7.792034628 .0397055793$ H $6.2685723101-8.992943669997 .5025877363$ O $0.1703455349-11.88879910980 .8770117858$ H $0.8763300524-12.20786678440 .2703769795$ H $-0.6010267071-11.80782724130 .2763093324$ O $-2.6666488-5.2225490829-12.6147935008$ H - $2.9254867632-5.3671829374-13.5266527807$ H - $2.9565545114-4.2961899995-12.4528309497$ O 0.69266167686 .838273314612 .1715219166 H 1.48116518036 .88322543511 .6149524225 H 0.80935315495 .997470230112 .6558517676 O -7.2396000511 -7.2982019431-2.9221774687 H $-6.4324132331-6.8619752588-2.5869446978$ H - $7.0467624408-7.4066032747-3.8752874175$ O $-2.7380589709-9.08745969633 .8637995336$ H - $2.4067232985-8.21192399954 .157539704$ H - $2.0449562124-9.6877284744 .2166769333$ O $8.4475676481-7.11614534612 .268577047$ H $8.0750837585-6.35840302322 .7542969863$ H $7.6520485995-7.63850503932 .0284749904$ 
O 3.29995801714 .4230874659 .8967723886 H 4.22993823884 .45322507979 .5904721491 H 3.0370143435 .36612071249 .8845779096 O $4.4643716783-0.88869842759 .977012697$ H $5.1850277233-0.72657971079 .3284421079$ H $4.4305901469-1.87921278019 .9568568224$ O -8.5702277624 -6.1699757078 1.8503231312 H -7.7660823358 -6.4598349433 1.3659571897 H -9.008205632 -7.030427756 2.0445981334 O - 11.16241315710 .88614573110 .4855507286 H - 10.18906869890 .72834091650 .4146706569 H - 11.21271769021 .85576820040 .5237320985 O -2.90185695084.79749984159.3765497692 H -3.4773446047 4.07925562989 .7119262023 H - 2.71447991484 .458937018 .4667569141 O $1.10602125211 .3536813494-7.8913532938$ H $1.52379028032 .1892136963-7.5828350856$ H $1.74475033570 .6770804724-7.5732099153$ O $8.9852137707-2.0590198355 .7139908253$ H $9.2946245455-1.14363391465 .5387586228$ H 9.285690493 -2.5347856294 4.9094827231 O -13.57034947332.86255860912.3910026704 H -12.8356404677 3.15308469791 .8181023362 H -13.4472747835 3.43671621123 .1820777875 O $-10.82912234137 .1657136305-4.6670954134$ H - $10.19142989237 .8947987626-4.7143204682$ H - $10.88478629226 .8562131922-5.5902971127$ O - 11.1984594970 .29789047718 .1375081467 H - $10.3200378583-0.07812162357 .8835317871$ H - $11.7746243994-0.47281193628 .0730592311$ O $10.65300622212 .1569774081-5.2590947257$ H $9.70848036162 .2489035815-5.0519999203$ H $10.65834566061 .400773051-5.8876628716$ O $-2.12196228286 .4111545898-6.473710978$ H - 2.7009870522 $6.0598098912-7.1815416336$ H - $1.90154733287 .3094724674-6.815734946$ O 0.84874291054 .283494071113 .06116117 H -0.05918179964 .056995701812 .7906401175$ H 1.38269466633 .605945707212 .6139993801 O 2.35316320032 .523178995711 .5316491588 H 3.12717690362 .109488201111 .9248517685 H 2.73487671423 .215197363210 .9461516307 O $7.6897157403-9.34726980434 .9475383764$ H $7.4152539795-9.84701453315 .7378943526$ H $6.8307049175-8.99611628514 .6343976968$ O $12.0025785911-5.51926427151 .6901937128$ H $12.1496049861-4.92220989650 .9303279926$ H $12.2274097657-4.94478629352 .443614567$ O -3.3436286282 -10.9520345309-5.1852442854 H -2.9452274864 -10.6789630887 -6.0306127646 H $-3.7163930519-10.1203125282-4.8540188815$ O $4.67266574958 .6223533193-2.2035150036$ H $5.08445699827 .8337122237-2.6302263202$ H $5.33086525718 .835164433-1.4974888513$ O $3.1583851752-2.2925437007-11.8402900838$ H $3.3007509557-3.2145051343-12.1342913139$ H $2.4673765759-1.9879320328-12.4669202056$ O -3.315657931 13.1618932947-2.9020461886 H -3.0189674092 $12.4467695321-3.5006072951$ H -3.7481950115 $13.7671480394-3.5209868727$ O 3.748603315512 .21900095874 .20001206 H 4.409060090812 .37867345963 .5088432248 H 2.915192499812 .33792041463 .7124352982 O $12.30809274631 .9594246508-3.1115924758$ 
H $11.87817502312 .2519233911-3.9390771536$ H $11.66916860341 .2878248706-2.7926938163$ O $-1.8419261238-7.1550275348-8.2606161074$ H $-1.6233373377-6.9467865936-9.1964739329$ H - $1.2022078618-6.5962511427-7.7700808988$ O $9.720807156-7.57783967294 .6948343519$ H $9.1269486263-8.3273096774 .8906954846$ H $9.454887736-7.34594631443 .7901634434$ O $-3.602135462-12.1135257835-2.7164969219$ H -4.3726894581 -11.5769511116 -2.4555050128 H -3.4799490904 -11.867954979 -3.6576466209 O $1.2261175921-9.35827175163 .8642197454$ H $1.7032355751-9.07765727083 .0553287447$ H $1.8797564252-9.16378227284 .5601731337$ O $2.7337776847-11.49237379432 .188826763$ H $1.8171579695-11.69980534931 .9662055512$ H $2.8025648559-10.54532573781 .9658190445$ O $11.50911702835 .4881720817-1.9973108889$ H $11.29227905996 .0913747875-1.2691549209$ H $11.76662824644 .6764934328-1.5331758558$ O $-9.0344494241-4.3643736308-4.5984361983$ H -9.6451474954 -5.128700087 -4.530384361 H -8.9004150986 -4.1356536928 -3.6706953864 O -6.29840554984 .03853452358 .6155254091$ H -5.54677371113.57360068419.0424788609 H -6.3057179792 3.62846808127 .7257252698 O $-5.8811412933-1.8625283442-7.6769763926$ H -6.0592414181 - $0.972874107-7.3057501551$ H -6.6271987916 -2.3982115574 -7.3235041353 O $-9.13799485693 .8465104426-2.9728879569$ H -9.5357726942 $4.6650602417-2.6046546471$ H -9.0630122835 4.0621411324 -3.9267797366 O $8.15409950446 .3243536969-2.5019028755$ H $7.1881945516 .4308519496-2.6484683588$ H $8.43043995945 .8811679059-3.3283542343$ O $-5.54810423619 .7244150516-7.1599594712$ H -4.8311846362 $9.9107485784-7.801589109$ H -5.0452634608 9.5222820306 -6.3336919929 O 1.557578657612 .36299810842 .5618650318 H 1.979323428512 .92844273531 .8944854555 H 0.617245521112 .39942704742 .2864413055 O $-5.3315341411-2.5440555115-10.2177543945$ H $-5.5248462038-2.1729559076-9.3287222418$ H -5.0633098098 -3.4588752252 - 10.0082227318 O $7.1358867496-5.4682925648-4.9997037641$ H $7.7680962091-6.1598591429-4.7196513735$ H $6.3831798621-6.0008042432-5.3324715068$ O -6.7855610030 .174017054610 .1922445992$ H -6.771517513 0.11879541559 .2260561239 H -7.2389818552 1.02207355310 .3555155456 O $-1.675737873913 .2947837961-0.733252504$ H -2.3229174923 $13.3040474683-1.4680968321$ H $-0.825431562213 .3806833007-1.1902556602$ O -2.41629075090 .386519254811 .2364561621$ H -3.2723844536 0.054077361911 .5674030535 H - $1.7665096331-0.087600341311 .7773986923$ O $-5.9208071339-8.08072408894 .4473893294$ H -5.5608908564 -7.1659255806 4.5072426748 H $-5.6363408299-8.46571437775 .2957526933$ O -7.1614693447 $3.4304351565-9.4971954565$ H -7.8602446615 3.2117080201 -8.8384425269 H -7.2865611997 2.7371305391-10.1722310197 O - 11.72227803343 .66854781460 .5084725403 H -12.19175437 $3.5337038623-0.3384780184$ 
H - 11.56358435194 .63661279660 .5016424078 O $8.1244199527-2.2199829114-7.3648317262$ H $7.783076548-1.5427310804-7.9909559489$ H $7.7783918817-1.8900757545-6.5066099898$ O $-5.4084835269-10.3170449108-1.6477478795$ H -5.960036897-10.6548132991 -0.9147886219 H -4.8165561034 -9.694583376 -1.1796407575 O $-1.2649571752-6.6238730228-10.8402859804$ H - $0.4219436215-6.1390946915-10.948378257$ H - $1.8541384716-6.1359463443-11.4663383726$ O $11.06843968756 .2746101886-4.5796582508$ H $11.50850520645 .6330654757-5.1890133736$ H $11.33553147885 .9433489735-3.6922515334$ O $-6.25849220398 .5096038867-0.6480865127$ H -6.2684704414 9.17549729240.0690679616 H -5.6734377686 7.8161967729 -0.2682816744 O $-7.798719432511 .3951395232-4.0059819703$ H -7.3121469958 11.8785357317-3.3256586127 H -7.4415176078 $11.7515704386-4.8379973125$ O $2.40528036958 .4716671097-5.5957147508$ H $1.62114849238 .1935164564-5.0729189707$ H $2.80844184599 .1309388308-4.9999119535$ O -3.0076562546-14.164514153-0.9729974627 H - 2.1641388867 - $14.4059091507-1.3828619741$ H -3.3840310165 - $13.5545864574-1.6334695741$ O - $1.0677320597-1.1803410771-12.2261684191$ H $-0.8036526448-1.6875806993-11.431134032$ H - $1.2550024723-0.2870864027-11.8622835824$ O $2.4577783558-4.60813187158 .7828991942$ H $1.5992418808-5.08104843088 .8529089823$ H $2.2088500279-3.82892351178 .2343516178$ O - $1.26357466481 .3801387328-11.3355343917$ H - $0.94956905072 .0998389654-11.9177744376$ H -2.0140524876 $1.790689951-10.880662221$ O 3.401364204311 .40795186326 .7241809668 H 4.329265816611 .210056436 .9215021423 H 3.471900417411 .75201758235 .8040887815 O $14.21979462520 .0496944494-3.2769864597$ H $15.087785020 .4211705702-3.4532337775$ H $13.63727474610 .8367904647-3.262403764$ O $-4.4839868112-7.0612566513-8.0454708491$ H $-4.7769449518-7.9788588893-7.9214264923$ H -3.5017477238 -7.142912425 -8.0402604468 O -5.59728218822 .369254928712 .3144984005$ H -5.2101064799 3.235622093312 .5361336351 H -6.4705957438 2.596614142111 .957607534 O 2.556434952913 .97800050750 .4951042772 H 2.492016785114 .93568318160 .4550514601 H $2.063033473913 .6921382452-0.2975695515$ O $8.90299576771 .6017169611-8.821975651$ H $8.34474316012 .1980840244-8.27115288$ H $8.30472555220 .8310122815-8.9517674041$ O -0.54329113171 .633776244513 .8891794392$ $\mathrm{H}-1.04597903212 .234074848913 .3112869434$ H -0.06355689432 .234907973814 .4642490677$ O $9.3261282103-3.7740165112-4.915544153$ H $9.9402695488-4.4455415683-5.2485364119$ H $8.4700431077-4.2414703932-4.9402916573$ O $-6.777357243112 .0032760262-6.4971501326$ H -6.4051202917 $11.1341905995-6.7663830519$ H -7.3948004306 12.2043410887-7.2044056448 O -3.4190597435 -2.6762658124-12.300587486 H -3.9211253562 -2.4023814711-11.5098922027 H - $2.6581393595-2.0630323901-12.3016857694$ 
O -11.9781613768 -5.0921736331 4.0827150526 H -12.7809609351 -4.8481900886 4.5890714766 H - $12.0359021891-4.48591129183 .3116340317$ O $-8.82605869024 .6592416375-5.5134623395$ H -8.1729088974 5.381234026 -5.3858238393 H -9.5427761537 5.1233060606 -5.9911875097 O - $2.1324301118-4.63244932349 .7312476951$ H -3.017431491 -4.871514961 10.0550066853 H - $1.8653301992-3.910688640610 .3480884379$ O -11.73587776041 .99995191276 .0300222455$ $\mathrm{H}-12.3063626891 .51830530165 .3858153104$ H - 11.74257706831 .40191312986 .8006627987 O -3.3677696794 10.60641425371.9823949704 H -2.553299324510 .12892894412 .2080551878$ H -3.2754841912 11.43597167432 .4931302713 O $2.3927215196-6.342092769-9.0426222985$ H $2.4366842173-7.3291921691-8.9671628097$ H $2.4176145807-6.0655980862-8.0995045126$ O $2.46066910983 .5374370897-7.0794772909$ H $3.39843376183 .7860962767-6.9853883021$ H $2.08781441883 .7901371723-6.2073620821$ O $6.2627178146-8.60355402741 .7368505006$ H $5.8170043496-8.6460264382 .6123456382$ H 6.3673999142 -9.5498293918 1.5153081068 O 10.77042760131 .19345527910 .8586286357 H 9.89343310271 .09866223861 .2782237395 H 11.37067493261 .21208293121 .6219689985 O $-8.41523345075 .9106894018-8.7827359345$ H -7.9777911464 $5.1723355732-9.2328935615$ H -7.6670552956 $6.4079157905-8.40740921$ O -7.2128739553 1.1875085569-11.1000953577 H -6.2934585037 $0.9541709032-10.8498955649$ H -7.1453964411 1.2898588238-12.0568220487 O -9.72339124445 .61740520923 .4644901349$ H - 10.42565842865 .83938873394 .1003803356 H -9.4056657775 6.5046544156 3.1835049123 O -9.673736651 -3.923901553 0.9243785091 H -9.3777760632 -4.7882918544 1.2945632656 H - $10.5721088009-3.82839669981 .3081548527$ O $6.4668755143-0.60240560348 .2597861009$ H $7.2724217059-0.77799761868 .7820251252$ H $6.487004819-1.31105902517 .581193121$ O 5.52429067491 .84782791037 .8079439798 H 5.91112442150 .95216271517 .9272723972 H 4.56581262951 .65441679947 .8964073085 O $1.0792731511 .4664480792-3.9892574749$ H $1.9725064911 .0628336071-3.9147678057$ H $0.538124649710 .9269773689-3.3862928005$ O - $2.601289958711 .2905854098-4.7533225235$ H -3.1418817331 $10.4922702005-4.9561256722$ H - 1.869686277911 .2324808558 -5.4015946846 O $10.3325768377-7.37576626410 .3848775852$ H $9.611835828-7.23843458641 .036368034$ H $11.0116522349-6.75457932720 .7015025493$ O -8.9950668707 -3.3206210241 7.139419235 H -8.4265608251 -3.8060996636 7.7674631229 H -9.2283750642 -4.0246175771 6.4978702381 O - $12.0507958581-7.36024352912 .4601982088$ H - $12.1334082621-6.94749021591 .5836888579$ H - $12.1283325864-6.60711687083 .0703355223$ O $2.7301955934-0.654402025712 .2184226683$ H $3.4404016638-0.886662988912 .8374266668$ H $3.1731300738-0.712982637111 .3590708156$ O $12.531563444-3.78692752143 .7862039903$ 
H $12.5786952679-2.81223278013 .8803958603$ H $13.1952417138-4.09965710794 .407836078$ O $5.4660844471-3.8662565693-8.2923290557$ H $5.2917051579-3.655714318-7.3516116279$ H $6.3819244974-4.2012722122-8.2553798926$ O - $2.2846204335-9.7657717834-7.4725104073$ H $-1.542689397-10.1877547856-7.9190687221$ H - $2.1338846514-8.8200894404-7.6530998455$ O $12.2452622777-3.669457328-0.3204136454$ H $12.0833299581-4.1096036956-1.1732863096$ H $12.6104784721-2.8091462332-0.5970269159$ O -3.4288364812 -13.4477042454 1.5882976926 H - $4.3596662404-13.64729855361 .7577526218$ H -3.2913995814 -13.79346897360.675729809 O $5.0431387848-6.8328182222-6.0755524453$ H $5.4657145743-7.0803541379-6.918506329$ H $4.5400260532-7.6381416342-5.8276906262$ O 2.67287643999 .39791218488 .4549382967 H 2.7580458501 10.1991417875 7.9025345731 H 3.28359482748 .78754887217 .9901617393 O $12.864777052-1.0986616287-1.0784071678$ H $13.4152923009-0.9255904584-1.8606084214$ H $13.2266732958-0.4670664309-0.430274532$ O -6.8651173303 -10.889502949 0.6319783403 H -6.4226938446 -10.182512838 1.1501331994 H -6.7163257613 -11.6729053046 1.1962369159 O $7.9065546875-5.075199036510 .7882874662$ H $8.591977691-4.373084932310 .6846991641$ H $8.4067202944-5.818210894911 .1343816548$ O 4.32978301697 .91199031376 .9776760937 H 3.72601304467 .8587983426 .2060496691 H 4.83749514197 .06633202436 .8934993476 O 7.696563115610 .66097556181 .5086042915 H 7.44626748110 .78658336152 .4462884644 H 7.376059228211 .46937814691 .0739823555 O $-4.235041799-2.23078423298 .9058752191$ H -4.4264229933 -3.0241700958 8.3877621221 H -4.2576038536 - 2.548828407 9.8232352085 O $-9.6204442412-5.27204372815 .3297660392$ H - $-10.5141337879-5.19337185424 .9158045125$ H -9.4188094335 -6.2179385231 5.2002772341 O $11.24479999143 .1244901861-8.7471450668$ H $10.40872229952 .6317129754-8.7995643619$ H $11.80275483992 .6916680456-9.3946083343$ O $-2.5942260096 .1246204842-10.2444925526$ H -3.3634614977 $5.9003159284-9.6896603391$ H -2.62143444 5.4458536192 -10.9624690362 O -8.07023931554 .38980138865 .2885244817$ H -8.7416345726 4.25152875695.986062033 H -8.6105262346 4.7294755204 4.5498183059 O $6.47945156912 .242135255-0.5080352333$ H $5.613515148412 .124810623-0.960370614$ H $7.10414909411 .9353845056-1.1869345061$ O $-1.3297724963-2.501217895911 .0892522034$ H $-0.847752458-2.025032737611 .8001602338$ H - $0.9600744452-2.066747876410 .2918075184$ O 5.72730859815 .70728142756 .7948903711 H 5.79208244435 .19957277687 .6344046648 H 5.76704093225 .00302750156 .1210927351 O $-0.8168657027-10.67593413684 .7659318233$ H -0.8917691115 - 11.4544059784 .1726999263 H - $0.0131243891-10.21290112494 .4045969642$ O $-1.5693396306-7.27954472127 .3143048276$ H - $2.4802455166-7.12787713827 .6426792912$ 
H - $1.4842635598-8.2611684977 .3500857434$

O $-6.4291192365-7.9759302866-5.4422882235$

H -5.6172987512 -8.2619218752 -4.9752969694

H -6.4396229778 -8.5650080662 -6.2214345882

O - 2.894747295512 .90502165583 .3791138453

H -3.5401892278 13.60357445943 .5205350127

H - 2.490379721112 .80104437734 .2789777629

O $3.69198894440 .4456676722-11.4848307364$

H $2.78163308170 .528403821-11.1211149408$

H $3.7693479506-0.510687678-11.631488516$

O 0.537348383911 .39615740956 .6991386281

H 0.659312597810 .86491006385 .888631793

H 1.448259021211 .67147011856 .8902825699

O $7.98776586922 .7156700889-4.5079851581$

H $8.20111959453 .6784157393-4.5742794306$

H $8.2426205572 .5036271557-3.582090574$

O $0.2774448493-11.1946434194-2.4364505341$

H - $0.5057594283-11.1722672673-1.8423111366$

H $0.9712138241-11.5896285618-1.8705813891$

O $6.4243188885-10.81901598826 .9014239652$

H $5.5036435047-11.05464741026 .6905007467$

H $6.9095316612-11.58611092526 .5504262394$

O $3.497647896810 .3042778167-3.8808256038$

H $3.95989728939 .7012627222-3.2492025088$

H $4.189901988510 .4430061185-4.576322746$

O - 12.30329480358 .87732016330 .7895690646

H - 13.16916473048 .7804377631 .187475477

H - 12.02764152497 .96683729770 .610328265

O 1.257403781110 .450839475210 .5118821008

H 1.900993371811 .017423227910 .9432737947

H 1.77807037610 .02053434399 .8031414845

O $4.9420833126-12.27549983023 .4565400462$

H $4.1103559799-12.11693396152 .9578388697$

H $4.6707717261-12.0588281014 .3639740732$

O $-4.9263748979-0.330558174312 .0364125393$

H - $5.6338576721-0.297614348411 .3514321435$

H -5.0927218273 0.501888562312 .5119080366

O -9.53561520839 .62865611470 .6693597245$

H - 10.47944721039 .85302752310 .7158321981

H -9.098228947 10.49316600920 .497051404

O $6.96313543137 .2861108102-5.6576527849$

H $7.21704148738 .2210241259-5.5338094941$

H $6.12002775797 .3504384105-6.1608099177$

O $3.031302922-8.91070644055 .8383018339$

H $3.1048899569-7.93444418595 .9545039151$

H $2.4075134862-9.14357680246 .5746449673$

O $-9.4555132715-7.1617082357-1.4612340804$

H $-9.3864864848-7.9660566296-0.9116535934$

H -8.6192702902 -7.2009143044-1.9814640038

O 10.43069009262 .76942653064 .0882746444

H 10.82151395463 .42999653654 .7020205866 H 9.73853852133 .30274700463 .6530432248 O $-5.22480625760 .9541659162-13.3231131944$ H - $5.38069165580 .0385113461-13.6253501119$ H -4.9137582723 0.8294215928 - 12.4079702136 O -9.0976159641 -9.5991384639-0.1611394241 H -9.1239768804 -9.9183906241-1.0782981237 H -8.3262700912 - 10.09200808090 .1900259411 O - $-1.2870178883-12.68996580293 .0292109899$ H - $2.0628181277-13.02798852062 .5280260456$ H $-0.6618256326-12.47883521122 .3068994916$ O $0.8422832729-9.6958275866-6.1853405172$ H $0.1568025527-9.0681627855-5.8614722869$ H $0.5436956854-9.8930010863-7.0895918902$ 
O -12.1474233304 -3.4514866951 1.9657891762 H - $12.7368312828-3.45899784591 .1844793726$ H - $12.1471791973-2.5025401712 .2070484993$ O $-8.7758920812 .8400622993-7.4996293783$ H -8.7299654784 $3.439057265-6.7244681967$ H -8.8800764477 $1.9589307558-7.092139584$ O $-1.2698097444 .2980989278-8.7028841321$ H - $1.69250550695 .0431006172-9.1738899983$ H $-0.31819958084 .4727013971-8.8859198827$ O $5.00571604074 .5193051683-6.5559766038$ H $4.85700126075 .4920099709-6.6047057647$ H $4.8471526174 .3441346643-5.605332599$ O $4.3355693052-8.5774774454-1.866122773$ H $5.138112733-8.6568414929-2.4298239735$ H $3.7008311846-8.1280742462-2.4525548037$ O 12.51010870771 .36285396113 .0465046736 H 13.27492228321 .9104348813 .3075389287 H 11.76281008631 .86500539393 .4356537656 O $4.071546711111 .7917557-1.5658887318$ H $3.260370474711 .3905791598-1.2000662589$ H $3.987834964111 .5820962045-2.5069319145$ O $10.622548638810 .7424453387-0.6428090448$ H 10.700721122310 .48055875350 .2810187589 H $10.36987894289 .9231627675-1.0883622048$ O 6.24552051020 .424962683211 .6409729882 H 6.0366945310 .015231794412 .4961687609 H 5.54944705610 .055342938811 .0679179593 O $-4.1104672493-6.64228337448 .0947209759$ H -4.2753045673 -6.3779386448 9.0174589726 H $-4.3348771865-5.81377237397 .6166538136$ O $-7.845851626-3.5061854012-6.8256595803$ H -7.5010373506 -4.3851643866 -7.1017543905 H -8.2727858347 -3.7367888403 -5.9652960036 O $5.05772310162 .4704236791-10.3899654136$ H $5.97163285222 .4492422814-10.7451821965$ H $4.66408740321 .6624147546-10.7938561946$ O $3.8761605778-7.03453094218 .8435201471$ H $3.5588440245-6.13776558419 .0380163997$ H $3.2432045854-7.58826619869 .3280667928$ O $9.45511257328 .5632022041-2.0473170112$ H $9.85474965028 .6716596898-2.9311519394$ H $8.92252268467 .7382874246-2.1618388114$ O -2.4115203586 4.2274416663 -12.0916317441 H - $1.53796790634 .0599490038-12.4819049357$ H -3.0229504302 $3.9205890541-12.7959145962$ O 10.13134450310 .30796423055 .1857263902 H 10.50860287270 .55299157366 .0527295631 H 10.10314625521 .17776179994 .7329502278 O 7.240180351710 .34326401214 .2206936028 H 8.05279287549.81755497874.4009447239 H 6.921773407810 .55126366865 .1161622119 O $12.3782086997-1.10072651274 .2670393599$ H $11.5001598339-0.80411390224 .5620848997$ H $12.6429566352-0.35943776633 .6904909584$ O 10.69954171235 .56966477212 .2129286365 H 10.51999324936 .05990772231 .3856919136 H 9.82310117075 .193832762 .4420468725 O $11.8249930514 .3747649686-6.2757818519$ H $11.76548844444 .1760678312-7.2245539766$ H $11.46201968863 .5607730331-5.8780899867$ O 7.89445152882 .47681945366 .7421884306 H 6.9969117422 .30308604267 .1041144868 H 7.71782942682 .40316268825 .7829636064 O -7.2061291266 -5.03541384178.4467780083 
H -7.4853861158 -5.9139150673 8.1323433838 H -6.4305334413 -4.8360657962 7.886470258 O -5.9020001467 12.2161168847 -1.9576341338 H -5.5694707661 $11.3366900359-2.199513362$ H -5.1202640049 $12.7736575017-2.0716628687$ O $12.12890771773 .0762977794-0.6096294866$ H $12.2606099292 .7283250871-1.5122113447$ H $11.52041120382 .4186625851-0.2113398202$ O $-9.6537759888-8.54801812682 .359871508$ H - $10.6068879801-8.31141755092 .4094839794$ H -9.6040747601 -9.0585902209 1.5282533995 O $-4.02069884363 .2485847623-14.0187556757$ H -4.3900883543 3.4782363674-14.8726537368 H -4.4774222566 2.4090709702 -13.7958987328 O - 12.88709184444 .21577223344 .595041165 H - 12.48698968715 .03617181834 .9399514755 H - 12.54630374923 .54255426275 .2004960564 O $-6.8912890042-5.9706210017-7.3238536555$ H $-5.9869073153-6.2114259667-7.6013785947$ H -7.0216368278 -6.5549953498 -6.5559728519 O -4.4966603516-11.1715836521 4.1130224838 H -3.9510948935 -10.3659003171 3.9871432466 H -3.8784910597 - 11.78050909554 .5639974808 O $1.0658404471-5.2164608249-11.0513739365$ H $1.5286316461-5.6205747558-10.2811452499$ H $1.7792484169-5.1333814361-11.7021974762$ O $-4.5863063799-5.1282522002-9.8868410182$ H - $4.58351227-5.8657526208-9.2406747703$ H -5.1857920215 -5.4569035073-10.5798124859 O $4.49707572737 .1443315343-6.6741457397$ H $4.41268725497 .3720088753-7.6285373973$ H $3.70762057627 .5886384335-6.2866011547$ O $5.9199351427-3.49049189712 .0789070842$ H $6.6492076892-4.087606819511 .852441652$ H $5.3798303806-3.511447961911 .2665025185$ O - 10.12739507023 .97190014827 .0607835203 H - 10.09297306233 .6691476467 .9839644189 H - 10.73508084253 .32715359216 .6533359354 O $4.6724291012-5.59555297-10.1710225791$ H $4.9332971259-4.9139238767-9.5170577907$ H 3.832024764 -5.9307456679 -9.7885975275 O $-5.5423963115-8.69718593127 .1282139837$ H -6.4225670641 -8.3522977308 7.3660431503 H -4.9467598529 -8.007123476 7.4944141347 O -7.82165966522 .703000452610 .4715332059$ H -7.3996479357 3.30474924299 .8295933251 H -8.7546281728 2.7131685674 10.19358004 O $1.2717565471-12.014279385-4.8438000156$ H $1.0964732297-11.2250452445-5.3899883901$ H $0.9289948276-11.7382351401-3.9760181459$ O -1.8664249664 12.5918415745 5.7725731729 H - 1.006503365512 .32069536866 .1352040411 H - 2.491516562812 .3119008126 .4686960576 O $1.27520422590 .9440459127-10.5133197253$ H $1.1710697111 .040168067-9.5408840605$ H $0.34692793080 .9970436847-10.8215288038$ O $0.0915115987-5.91728678778 .9104385118$ H - $0.6491392686-5.42035786189 .3171585738$ H $-0.3851447381-6.41372998368 .2078238662$ O $-10.6755072187-6.3871264086-3.8602218078$ H - 11.6135212611 -6.307085506 -3.6242044088 H - $10.3053244581-6.8634496131-3.1002687889$ O - $0.302128225810 .9854956433-6.2238525434$ H $0.189949427411 .2796458972-5.4232092768$ 
H $0.418375311110 .9203313669-6.877536005$ O $10.6740254715-2.67242275897 .8776065795$ H $11.5160495957-2.30077121067 .5778645702$ H $10.0747936176-2.51850198227 .1302877776$ O $-14.2263343143-4.15748759715 .3258990181$ H -15.1395992048 -4.3577320178 5.5349565193 H -14.155370871 -3.201917285 5.5147988945 O $3.2061051626-8.6641170994-5.3174685588$ H $2.9072294152-8.1101580708-4.5660783701$ H 2.3601950268 -9.0395550044-5.6391165002 O $1.01675974913 .0383440031-1.6283768229$ H $1.156943689512 .1273843122-1.3089467496$ H $1.061714111912 .9276128369-2.5896931466$ O $7.65236638362 .410534096-11.2101647888$ H $8.23610100252 .1727719794-10.4729355015$ H $8.25892157092 .6329679919-11.9188308483$ O -6.22701696626 .72753103188 .9666973836$ H -6.361145657 5.76927904168 .8075287879 H -5.5964743285 6.72446651759 .7061064318 O $7.3471085067-12.66286486884 .9960123864$ H $6.5608469187-12.80070445574 .4500131584$ H $7.9993923252-12.33900558624 .3576862928$ O $9.121629891-8.4604288948-1.856724567$ H $9.0099167389-9.3320211029-1.4223882314$ H $9.6093442049-7.9642779104-1.1699789895$ O - $13.3232567348-0.1748149411-0.7060204967$ $\mathrm{H}-12.51555433370 .1663545875-0.2646871723$ H - $13.99962415730 .4590100311-0.4179756339$ O -0.037157022 -0.714524109 12.6023375232 H -0.17508020280 .064992533313 .1767485605$ H $0.9327812991-0.710045483812 .4783219905$ O - $1.66861275328 .9159272075-7.2850209607$ H - $1.18290917669 .6289352286-6.821275186$ H - 2.377401933 9.4054221116 -7.7411864402 O 13.00695111254 .28559039161 .7128223257 H 12.8014976853 .95107651170 .820791819 H 12.19206833034 .78325542211 .9357768423 O 5.84118626734 .19616632679 .0070327448 H 5.69883598653 .34125024598 .5423318266 H 6.46002524043 .92484046979 .7161326333 O $8.0558749548-4.7874324553-8.1421281421$ H $8.1261087702-3.8626869441-7.8176830651$ H $8.992725459-5.0568910537-8.1748279909$ O $5.5398783202-2.0588953142-10.3406427978$ H $4.7865631797-2.2210354079-10.9263216557$ H $5.43758473-2.7362692369-9.6457371961$ O -10.9918780337 $1.9192586422-2.9671490087$ H - $10.24000990762 .5482387891-2.886784057$ H - $11.697639382 .3657402689-2.4711870751$ O $2.1199423179-12.4233332035-0.8653979101$ H 2.4579187599-13.2946910068 - 1.0934440527 H $2.9550352412-11.9031320828-0.7280272748$ O -8.0569113605 -10.0226760292 -2.7379766978 H $-7.9053834197-9.1072392888-3.0073214721$ H -7.1630736255-10.3498940801-2.5756765354 O - 10.42628401182 .54384966429 .4637216215 $\mathrm{H}-11.12315506192 .710251585210 .1040892501$ H - 10.70636211911 .70153021459 .0469864279 O -14.8734047179 1.96853233310 .231126498 H - 15.79884823961 .86869052590 .4697698055 H - 14.4619316072 .27331703251 .0728212318 O $12.8903308058-2.2323492862-4.161518373$ H $13.4878913284-1.483270052-4.0064401613$ H $12.0778786311-1.8075315807-4.4768494702$ 
O -1.0145337355 -9.2931906135 -10.8982179106 H - $1.0856539716-8.3138982958-10.8666805468$ H - $1.5774989368-9.5253696258-11.6390592568$ O - $10.58853524445 .9559223355-7.130870107$ H - $11.15979143135 .2257436315-7.4180070121$ H -9.9304696789 $6.0202687065-7.855522103$ O $8.6842202316-11.07559325623 .057938164$ H 8.4452827174-10.40509321113.7255807576 H $9.47831852-10.69023827032 .6370479799$ O $10.765924179-2.3950973741-7.9106831332$ H $9.8081740444-2.409326991-7.7232162464$ H $10.9757060365-3.3335092714-8.020010154$ O $2.6047203007-8.9474316442-8.7920440331$ H $3.2671832526-9.48885784-8.3218955067$ H $1.823435067-9.521568379-8.8018986529$ O $10.7781569836-5.2544471812-7.933558697$ H $11.3727271907-5.812747658-8.4400612141$ H $10.9576391442-5.5242193855-7.0046083735$ O 10.59939222497 .2641408510 .0609561408 H $10.16492971317 .6915725983-0.6989815121$ H 10.49109851317 .93839325830 .7561398557 O $9.6234815097-3.124699014110 .3364699745$ H $10.109413016-3.0512826039 .4917272976$ H $9.2389504348-2.235334497410 .4283097521$ O -7.2822330963 -3.0843010997-11.9551068619 H -6.5576057132 -2.8206078946-11.348222141 H -7.9974793166 -2.4782909647 - 11.6746349222 O $-6.1008412265-12.67475937772 .5733040666$ H -5.5360465058 -12.1115145527 3.1465307815 H -6.68031379-13.1108024134 3.2036701707 O -4.24496563774 .787357889612 .6028481121$ H -4.1275440674 5.2369431175 13.4436097786 H -4.2827399331 5.525924706811 .9618714091 O -0.73416656549 .175995735712 .1132009104$ H - 0.03924967299 .714589033511 .7092200827 H - 0.27807838748 .334044244112 .2914110074 O - $11.8920114071-0.6647613555-2.9916884408$ H - $11.40246607320 .1768162802-2.9705183444$ H - $12.5224630593-0.5514957143-2.2568353293$ O $-8.612683053-7.80587438924 .7947622942$ H -7.6721491475 -7.9574931247 4.5683008898 H -9.0690791841 -8.1996111154 4.0226585741 O -4.09699704716 .772631439810 .7448027883$ H -3.5967001646 6.1523985754 10.1755838848 H -3.6078907088 7.6130092079 10.6290703724 O 6.01356223058 .51590873639 .2631290075 H 5.95334960047 .89418701939 .9893791504 H 5.36417381368 .1952834358 .626140638 O $-6.2564241762-9.2828334393-7.8579032247$ H -6.3318321141 -10.1948098171 -8.149919651 H -6.7113165541 -8.7805912054-8.5636313293 O -6.16002031577 .64643355432 .4264181213$ H -6.0873096562 7.10089516123.2450364206 H -5.5594074449 7.1873233423 1.8059254738 O $6.4464705757-7.0210693777-8.4862295792$ H $5.9331939567-6.7174363386-9.2530682907$ H $7.0928121805-6.2976735074-8.3626737197$ O -1.014917948510 .70873614718 .8756792886$ H - 0.319230324210 .70960202859 .5535744276 H -0.515150777910 .93463916428 .0678688314$ O -8.80761128348.01622503762.7311059639 H -9.08343749248.6443485903 2.0317575608 H -7.8431950661 7.93323854962.570510551 O $1.1950812427-1.5135475334-13.5739955629$ 
H $0.9362599308-1.8951735404-14.4166602324$ H $0.3302839582-1.3893260884-13.1163716779$ O $-2.39146661438 .7669207668-10.6420098623$ H -2.4919349335 $7.7914926373-10.5870808919$ H - $-1.43112355318 .8800942011-10.6048272788$ O $9.0787052304-7.3641856162-4.3391309214$ H $8.8446781737-8.2067641106-4.7791447283$ H $9.1680698359-7.6509648778-3.4042898794$ O $5.139364367-1.375448178313 .5243819244$ H 5.2518998707 -1.6293625668 14.4430990593 H $5.4415437801-2.173894025713 .0350349274$ O $3.9123956609-11.46057210425 .9423796949$ H $3.591674194-10.54272458675 .8331834546$ H $3.1512141509-11.91464983356 .3145985602$ O $-5.5988229216-1.7262866724-13.7754829537$ H $-4.7961302179-2.1482060188-13.4317163922$ H -6.3099777828 -2.2309065972 -13.352410593 O -5.977674732110 .16907467061 .4832558324$ H -6.0909220225 9.349539717 2.0052401025 H -5.0499104278 10.41070907421 .6804637507 O 9.97339233019 .25942321961 .8596094026 H 9.15966308469 .76463179141 .6601544036 H 9.86595076569 .05817606412 .7990057023 O $0.2012928583-10.4324010795-8.8182979765$ H $-0.2175804522-10.0008627115-9.5995946069$ H $0.2853570803-11.3486286431-9.097336322$ O $1.3437805612-9.5214004547 .7468316356$ H $1.37586182-9.12950844558 .6339950013$ H $0.3904907022-9.72274623817 .6328507444$ O - $1.2857335438-9.92663521287 .2491975492$ H - $1.118563758-10.27022121366 .3362036686$ H - $2.1444387365-10.35186405437 .4657566226$ O $7.32934763943 .0687109479-7.2190055071$ H $7.62554581392 .9083686231-6.3080098688$ H $6.56580335983 .6550861743-7.0880708623$ O $12.1365275225-7.95980027833 .1715319596$ H $11.5147545904-7.75811228283 .8843864777$ H $12.1857101806-7.1322186972 .6689570031$ O $11.7314709424-4.3690928368-3.0097197855$ H 12.2600227785 -3.6672997704 -3.4439107755 H $10.824080232-4.1132369875-3.2093703676$ O $8.57500827445 .2616891581-4.9237141897$ H 8.0379305828 5.9524103414 -5.3740823592 H 9.48434366655 .6194249248 -4.9972466202 O 11.35104510724 .69966946525 .7280480682 H 11.4570114345 .60343699285 .3755535971 H 10.65419645664 .81054206626 .4055989533 O 14.45568980093 .21551442673 .6272466409 H 15.36524109553 .49646466983 .5018882916 H 13.97233529533 .70690843742 .9172868953 O $10.3877117388 .7985760214-4.6325881987$ H $10.64989481297 .8436120284-4.6767021781$ H $11.16031170729 .2445048965-4.9903775068$ O $-13.23597438233 .1002623972-1.6808988587$ H -13.9029427728 2.6542602395-1.1227715317 H - $13.76689272253 .5506212026-2.3441278783$ O $8.26827697211 .0584899937-2.3414154508$ H $8.457392622710 .1343633198-2.1039391983$ H $9.054238690611 .5163404072-2.0144424115$ O $1.29046245634 .7970091567-9.1875261977$ H $1.730249564 .3309378193-9.9320203387$ H $1.74271596244 .416392524-8.4092535643$ O $1.954698048510 .1898078699-7.6743988079$ H $2.853522130310 .3904805831-7.9803064736$ 
H $2.11214605219 .5233215777-6.9776837532$ O $6.5169216107-11.29882510811 .450632127$ H $7.3394259849-11.32847349561 .9795703812$ H $5.8886760333-11.75362496632 .0492048901$ O -11.28099165186 .14787837355 .7031535817$ H -10.8739086209 5.5681067492 6.3674182492 H - 10.73039275256 .95738864265 .7593172078 O $0.1481900173 .2458521593-12.7970396514$ H $0.87020844983 .6716293633-12.3107530219$ H $0.63014718482 .6238344576-13.370149059$ O $-3.49730483110 .3220014838-8.8180513165$ H -3.4654451284 $11.2020292064-9.2038538731$ H -3.1898637842 9.7496490599 -9.5630156 O $-4.065025531-2.862320074711 .6132190835$ H -4.4327188763 -2.0144458811 11.938205911 H -3.1060548078 -2.7092618237 11.6527935699 O - 2.74474174569 .080224886810 .2969536704 H -2.21090122879.5961581229.6652391088 H -2.18594484349.126460698511.0992677146 O 13.88245449320 .92867334410 .5212251726 H 13.68850656821 .79288308870 .1418465225 H 13.56097665551 .00422386751 .427749479 O -6.22521241016 .32251313314 .7258696878$ H -6.8057429303 5.57437297364 .9582521006 H -6.3997760894 6.9585780068 5.4429678884 O $-4.558200832313 .4367413673-5.457390257$ H -5.3755447358 $13.0783900646-5.8331481954$ H -3.9458933087 $12.6942980415-5.493765645$ O 9.30997302898 .66246478514 .6308799774 H 8.82310109398 .03504384685 .2145872586 H 10.19712365068 .27110794344 .6245594958 O $3.5286672413-4.9220329108-12.4934505473$ H $3.9078127321-5.4358855404-13.2109425784$ H $4.0452432103-5.2170520952-11.7114740051$ O $-9.3223680205-4.3033915806-1.6964742521$ H -9.4171821157 -5.2693653717 -1.6878797365 H $-9.4908047164-4.0708140723-0.7562073596$ O $8.4176499596-0.643111052310 .2262770717$ H $7.7879042328-0.274624262910 .8687147329$ H 8.85561966880 .14781279169 .863290606 O - $0.6844445704-14.0165771563-2.6954372332$ H - $0.4315731325-13.0922808357-2.7968671238$ H - $0.5639968642-14.3798914269-3.5748683123$ O 5.986711958110 .13302762616 .7888596308 H 6.453981151410 .00925993427 .623788704 H 5.42581923829 .33819980236 .7425960849 O $4.3482564755-11.0472102767-0.6034747292$ H $5.1460675049-11.077848942-0.0559883858$ H $4.3989303621-10.1713440092-1.0316047081$ O $5.286896138810 .4219939541-5.7957179505$ H $5.186735041310 .398993135-6.7647930453$ H $6.245738779910 .3227243803-5.6627385405$ O $-6.4639874446-11.25806286136 .0439589219$ H $-5.8579774474-11.21393130345 .2878908172$ H $-6.3810985589-10.38951258986 .4488342297$ O $11.0837706726-5.9120220576-5.3649497123$ H $11.6090279901-5.6944611971-4.57967185$ H $10.4000796094-6.5177457041-5.0038785495$ O $-9.4911599893-1.8026187835-8.0301197128$ H -8.8759585354 -2.4545393891-7.6223502494 H -10.2703624438 -2.3317961649 -8.2192395596 O $-4.8975220556-5.141309977210 .2896330313$ H -5.820928104 -5.0605189511 10.0030908055 H -4.8158017109 -4.4482475425 10.9686097365 
O 9.24622961124 .66501865237 .471730826 H 9.2709907214 .56365505988 .4281334838 H 8.74102539653 .87212546857 .1804554003 O -8.7611542196-1.1524430704-10.7261092007 H $-9.0253975046-1.2151049098-9.7969895664$ H -8.3517358783 -0.2742149614 - 10.7870012116 O $10.6693382612-9.80812410271 .6882071363$ H $11.3655670733-9.46191725582 .2733385529$ H $10.5563176816-9.06945341841 .0651961572$ O -7.8345996951 11.64446758160 .0903815779 H -7.3123492084 $12.0138785679-0.639267829$ H -7.1506990656 11.26479556730 .6718581756 O $13.0667050405-1.45382424786 .9201249949$ H $14.014067128-1.54521152657 .0432005724$ H $12.9671562274-1.33109243435 .9609912499$ O 8.14719407726 .87551684456 .2159046785 H 7.21858824786 .6217636586 .3582986049 H 8.62450114756 .17636583946 .6934954315 O 9.30405482941 .58807073448 .8831019627 H 10.18864405651 .40469621338 .517851993 H 8.80271930911 .85158491118 .0858978045 O $-8.0324568273-7.5149489767 .4237826208$ H -8.794642519 -7.8514825274 7.9040615981 H -8.30568071 -7.6117814976 6.4858897512 O $1.4939381873-7.937512566410 .0728222695$ H $1.1895969901-8.000271285310 .9812455651$ H 0.9765077693 -7.1901191905 9.7116406232 O 7.45436426812 .789223516310 .6714393467 H 7.05154389162 .037389018411 .1376203105 H 8.2075429042 .389677992810 .2107402079 O $5.16621114775 .0123609763-9.3508151617$ H $5.14918757564 .15916536-9.8198367188$ H $5.21924377534 .7476631447-8.4231664699$ O $-2.8560868734-13.04878168685 .2748352302$ H $-2.2155511932-13.04973268584 .5421954257$ H -2.4189317214 -13.5625740419 5.9559516792 O $-11.7661774163-6.3440943022-0.1335854262$ H -12.3702857402 -6.1826481301 -0.8709454894 $\mathrm{H}-10.9500618204-6.6250931827-0.576561883$ O $-7.36353811-7.5243456634-9.6562368676$ H -7.2069884469 -6.9662491442 -10.434412295 H -7.4968209851 -6.8776624425 -8.9478772452 O $2.46730921563 .3515404706-11.083262058$ H $2.09794625972 .455598265-10.9771330505$ H $3.42197535093 .2052105863-10.9715049516$ O $7.85649143289 .8282108054-4.932545383$ H $8.78116487269 .512926059-4.937148461$ H $7.83856628410 .3989518474-4.1460164745$ O -6.83885232288 .12127184136 .7281691094$ H -6.7183948091 7.58439266397.5390325834 H - 6.39635859138 .94680426356 .9591818616 O $1.89187646031 .2501626306-13.7449941485$ H $2.717543081 .2427076484-13.2483441622$ H $1.65168857750 .3138612877-13.7868408988$ O $-11.53207187711 .0409480596-5.4897134097$ $\mathrm{H}-11.33315213531 .4622021948-4.6311492149$ $\mathrm{H}-12.07811147410 .2898560117-5.2314924413$ O 11.61256527747 .04778093364 .2635891933 H 12.40494440747 .48068373313 .9360325612 H 11.29967638296 .53438422543 .4876442202 O $4.40552661697 .6222475142-9.2984788798$ H $4.8187025076 .753605389-9.4928954458$ H $3.53876619347 .5292616734-9.7648812952$ O -13.486296989-2.9270942234-0.3557138914 
H -12.9581827876 -3.1654759566 -1.13251853

H $-13.5809713516-1.9641170317-0.4459616475$

O -9.0484704016 $0.3845840944-6.4719797912$

H $-9.92090178220 .5381218667-6.0632948559$

H $-9.2190310368-0.3926213504-7.0421192271$

O 11.58284416950 .99382242847 .4248665245

H 12.22100823221 .62909413047 .0349350005

H 12.12939538230 .21324121527 .5879185089

O $3.532398012-12.769320442-6.1398228767$

H $3.7249126573-13.665258986-5.8601265404$

H $2.7128703816-12.5528923455-5.6514652748$

O $6.6564583269-8.8278084254-3.1552844725$

H $7.4427790563-8.6393839171-2.6167571135$

H $7.0326267997-9.2590076853-3.9367529993$

O $-13.0966948356-5.7257209448-2.6110553716$

H -12.714583905 -4.8244231718 -2.649605471

$\mathrm{H}-14.0430476204-5.5665757848-2.6173047006$

O $-11.8464847612-3.3442030332-2.5778602489$

H -10.9147786087 -3.5658149589-2.4171172977

H -11.800794755 -2.414025568 - 2.880034141

O -3.4772850572 11.53333615677 .736475632

H -2.7331661252 11.29187175348.3065733943

H -4.1943564916 10.96103642148 .047315418

O -9.35567309878 .03550803695 .5051794063$

H -8.50245664248.1510444972 5.9574467612

H -9.1427853953 8.22884352594.5774340177

O $-11.59067550513 .2635511595-7.4020263731$

H -10.6983473918 3.0741662396 -7.7184078413

H $-11.77871356092 .521744315-6.8115605107$

O $-6.6798716091-5.6973533427-11.7012944952$

H $-6.7584294465-6.0486520581-12.5909767115$

H $-6.9840901689-4.76845681-11.8057797545$

O -3.8052519974 -10.8418681068 7.5498162213

H - $4.2967530857-11.61667091997 .25888436$

H -4.4543552034-10.1239691932 7.4598465558

O $6.9009670357-9.5656412169-7.6048767715$

H $6.7887200835-8.7013165013-8.0436032913$

H $5.9964307387-9.9269707297-7.5993259264$

O $8.7619379891-10.6688882763-0.3297676375$

H $9.4302738088-10.70483483580 .3694493914$

H $7.9756256163-11.04438179280 .0886593401$

O $0.14465953089 .0113785755-9.4008349194$

H $0.81164964689 .5329916045-8.9217581106$ H $-0.50105872958 .8021305627-8.707184148$ O $4.2997393131-10.3729960396-7.1771823287$ H $4.0083355567-9.8509667947-6.4073748919$ H $4.1133601181-11.2908556675-6.8913516692$ O -5.1226135189 9.4656487355 8.8597263394 H -5.64617671348.67749674649.0509861605 H -4.369888468 9.3807444515 9.4670700235 O $8.2511295557-9.766892078-5.3148703705$ H $7.7850722309-9.7037078293-6.1782387846$ H $8.6337327033-10.6474414252-5.3419198587$ O 13.18039945882 .70641681346 .0788334475 H 12.61143690553 .49794189646 .0397949068 H 13.79255352162 .85062348675 .3395643629 O $1.99882007727 .2117156209-10.2960060549$ H $1.63748555956 .3748552535-9.9533543045$ H $1.26317866317 .8349192271-10.1648777018$ O $4.711719964610 .1517553007-8.5039756295$ H $4.65548443199 .2271959738-8.8359232817$ H $5.046366365610 .6329156287-9.2649094287$ 